\title{
Temporal Contribution of Myeloid-Lineage TLR4 to the Transition to Chronic Pain: A Focus on Sex Differences
}

\author{
Nolan A. Huck, ${ }^{1}$ Janelle Siliezar-Doyle, ${ }^{1 *}{ }^{\oplus}$ Elena S. Haight, ${ }^{1 *}$ Ryosuke Ishida, ${ }^{1,2}$ Thomas E. Forman, ${ }^{1}$ \\ Shaogen Wu, ${ }^{1}$ Huaishuang Shen, ${ }^{1,3}$ Yoshinori Takemura, ${ }^{1,4}$ J. David Clark, ${ }^{1}$ and ${ }^{\oplus}$ Vivianne L. Tawfik $^{1}$ \\ ${ }^{1}$ Department of Anesthesiology, Perioperative and Pain Medicine, Stanford University, Stanford, California 94305, ${ }^{2}$ Department of Anesthesiology, \\ Shimane University, Shimane, Japan 690-0823, ${ }^{3}$ Department of Orthopaedic Surgery, The First Affiliated Hospital of Soochow University, Suzhou, \\ China 215000, and ${ }^{4}$ Department of Anesthesiology, University of Toyama, Toyama, Japan 930-8555
}

Complex regional pain syndrome (CRPS) is a chronic pain disorder with a clear acute-to-chronic transition. Preclinical studies demonstrate that toll-like receptor 4 (TLR4), expressed by myeloid-lineage cells, astrocytes, and neurons, mediates a sexdependent transition to chronic pain; however, evidence is lacking on which exact TLR4-expressing cells are responsible. We used complementary pharmacologic and transgenic approaches in mice to more specifically manipulate myeloid-lineage TLR4 and outline its contribution to the transition from acute-to-chronic CRPS based on three key variables: location (peripheral vs central), timing (prevention vs treatment), and sex (male vs female). We demonstrate that systemic TLR4 antagonism is more effective at improving chronic allodynia trajectory when administered at the time of injury (early) in the tibial fracture model of CRPS in both sexes. In order to clarify the contribution of myeloid-lineage cells peripherally (macrophages) or centrally (microglia), we rigorously characterize a novel spatiotemporal transgenic mouse line, Cx3CR1-Cre ${ }^{E R T 2-e Y F P} ; T L R 4^{f / f l}$ (TLR4 cKO) to specifically knock out TLR4 only in microglia and no other myeloid-lineage cells. Using this transgenic mouse, we find that early TLR4 cKO results in profound improvement in chronic, but not acute, allodynia in males, with a significant but less robust effect in females. In contrast, late TLR4 cKO results in partial improvement in allodynia in both sexes, suggesting that downstream cellular or molecular TLR4-independent events may have already been triggered. Overall, we find that the contribution of TLR4 is time- and microglia-dependent in both sexes; however, females also rely on peripheral myeloid-lineage (or other TLR4 expressing) cells to trigger chronic pain.

Key words: CRPS; microglia; myeloid; pain; sex differences; TLR4

Significance Statement

The contribution of myeloid cell TLR4 to sex-specific pain progression remains controversial. We used complementary pharmacologic and transgenic approaches to specifically manipulate TLR4 based on three key variables: location (peripheral vs central), timing (prevention vs treatment), and sex (male vs female). We discovered that microglial TLR4 contributes to early pain progression in males, and to a lesser extent in females. We further found that maintenance of chronic pain likely occurs through myeloid TLR4-independent mechanisms in both sexes. Together, we define a more nuanced contribution of this receptor to the acute-to-chronic pain transition in a mouse model of complex regional pain syndrome.

Received July 25, 2020; revised Feb. 23, 2021; accepted Apr. 2, 2021.

Author contributions: N.A.H., J.S.-D., E.S.H., R.I., T.E.F., S.W., H.S., Y.T., and V.L.T. performed research; N.A.H., E.S.H., R.I., T.E.F., S.W., H.S., Y.T., and V.L.T. analyzed data; N.A.H., J.S.-D., E.S.H., T.E.F., S.W., H.S., Y. T., and J.D.C. edited the paper; E.S.H. wrote the first draft of the paper; V.L.T. designed research; V.L.T. wrote the paper.

This work was supported in part by National Institutes of Health Grant K08NS094547 to V.L.T., Foundation for Anesthesia Education and Research Mentored Research Training Grant to V.L.T., Rita Allen Foundation to V.L.T., National Institutes of Health Grant R01NS094438 to J.D.C., and Veterans Affairs Merit Review 101 RX001475 to J.D.C. We thank Tian-Zhi Guo, Maral Tajerian, and Peyman Sabhaie for technical assistance; and Jasmine Dickinson and Gregory Corder for assistance with the analysis of affective-motivational behavioral data.

*J.S.-D. and E.S.H. contributed equally to this work.

The authors declare no competing financial interests.

Correspondence should be addressed to Vivianne L. Tawfik at vivianne@stanford.edu.

https://doi.org/10.1523/JNEUROSCI.1940-20.2021

Copyright $\odot 2021$ the authors

\section{Introduction}

Chronic pain affects 100 million people in the United States alone at an estimated cost of $\$ 635$ billion/year in medical treatment and lost productivity (Institute of Medicine, 2011). Pain is ultimately mediated by primary sensory neurons that undergo peripheral sensitization, and CNS neurons subject to central sensitization-induced neural plasticity. However, these neurons are heavily influenced by circulating and resident immune cells in their environment (Xanthos and Sandkuhler, 2014). This innate immune response involves, among other populations, myeloidlineage cells peripherally as inflammatory monocytes, and centrally as yolk sac-derived microglia. Several substances released from injured neurons have been postulated to contribute to 
microglial activation in pain states, including ATP acting on P2X receptors (Tsuda et al., 2003) and CSF1 signaling through CSF1R (Guan et al., 2016). Most controversial, however, has been the involvement of the damage-associated molecular pattern receptor, toll-like receptor 4 (TLR4). TLR4 is expressed by all myeloid lineage cells, and likely astrocytes and neurons as well (Ji et al., 2016), and was first suggested as a key trigger for microglial activation after genetic or RNAi-mediated knockdown resulted in decreased microglial activation and suppressed the development of neuropathic pain (Tanga et al., 2005). Subsequent work looking at limited time points suggested that TLR4 functionally contributes to chronic pain in a sex-specific manner, with modulation of this receptor only effective at improving pain in male preclinical pain models (Sorge et al., 2011, 2015). Importantly, however, other studies were not able to replicate this TLR4related sex difference (Agalave et al., 2014) or found it to depend on the paradigm and timing of TLR4 modulation (Woller et al., 2016). Previous work has been based either on systemically available TLR4 antagonists that affect peripheral and central myeloid lineage cells, or constitutive TLR4 KOs in which germline deletion or formation of a dominant negative receptor would affect all TLR4-expressing cells and carries the possibility of compensatory changes during development. In the current studies, we took advantage of a unique TLR4 KO mouse (Cx3CR1-Cre $e^{E R T 2-e Y F P}$; $T L R 4^{f l f l}$ ) generated to provide spatiotemporal control of gene knockdown to more precisely define the sex-specific contribution of myeloid-lineage TLR4 to chronic pain.

\section{Materials and Methods}

Animals. All procedures were approved by the Stanford University Administrative Panel on Laboratory Animal Care and the Veterans Affairs Palo Alto Health Care System Institutional Animal Care and Use Committee in accordance with American Veterinary Medical Association guidelines and the International Association for the Study of Pain. Male and female adult mice 10-12 weeks old at the start of the experiments were used and housed 2-5 per cage maintained on a $12 \mathrm{~h}$ light/dark cycle in a temperature-controlled environment with ad libitum access to food and water. Male mice weighed $\sim 25 \mathrm{~g}$ at the start of the study, and female mice weighed $\sim 20 \mathrm{~g}$ at the start of the study. Mice used in this study: WT C57BL/6J mice (Jax stock \#00664), Cx3CR1$C r e^{E R T 2-e Y F P}$ (Jax stock \#021160) (Parkhurst et al., 2013), B6.Cg-Gt

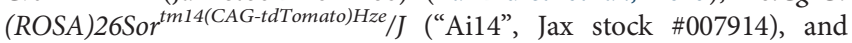
$T L R 4^{f l}$ (Jax stock \#024872). In order to confirm the expression pattern and tamoxifen dependence of the Cx3CR1-Cre $e^{E R T 2-E Y F P}$ line, we first crossed these mice to the Ail4 strain which has a loxP-flanked STOP cassette preventing transcription of the red fluorescent protein, tdTomato. To specifically and conditionally ablate TLR4 in myeloid lineage cells, we crossed mice bearing a conditional allele of the Tlr4 gene containing loxP sites flanking exon 3 generated previously (McAlees et al., 2015) with homozygous $C x 3 C R 1-C r e^{E R T 2-e Y F P}$ with subsequent backcrosses to generate CX3CR1-Cre ${ }^{E R T 2-e Y F P /+}$;TLR $4^{\text {fl/fl }}$ mice (TLR4 cKO). Mouse genotypes from tail biopsies were determined using real-time PCR with specific probes designed for each gene (Transnetyx). Control subjects for all behavioral studies were mice without the TLR $4^{\mathrm{f} / \mathrm{fl}}$ alleles: Cx3CR1-Cre $e^{E R T 2-e Y F P /+} ; T L R 4^{+/+}$that received tamoxifen with the same dosing and administration schedule as experimental mice. In some cases, Cx3CR1-Cre $e^{E R T 2-e Y F P /+} ; T L R 4^{f l f l}$ that did not receive tamoxifen were used as controls and no difference was seen between these and other littermate $\left(\right.$ Cx3CR1-Cre $e^{\text {ERT2-eYFP/+ }} ;$ TLR $\left.^{+/+}\right)$controls.

Drugs and route of administration. Tamoxifen (Sigma Millipore, T5648) was dissolved in corn oil at a concentration of $25 \mathrm{mg} / \mathrm{ml}$. Mice were dosed intraperitoneally with $100 \mathrm{mg} / \mathrm{kg}$ for $5 \mathrm{~d} ; 100 \mathrm{mg} / \mathrm{ml}$ TAK242 in DMSO (MedChem Express, HY-11109) was diluted to $0.5 \mathrm{mg} / \mathrm{ml}$ in sterile water. Mice were administered $3 \mathrm{mg} / \mathrm{kg}$ intraperitoneally TAK242 daily, or equivalent volume of $1 \%$ DMSO in water, for $14 \mathrm{~d}$ starting either at the time of surgery (early) or at 3 weeks after injury (late). The TLR4 antagonist, lipopolysaccharide (LPS) from Rhodobacter spheroides (LPS-rs) (Invivogen), was dissolved in sterile saline to a concentration of $2 \mu \mathrm{g} / \mu \mathrm{l}$ and $10 \mu \mathrm{g}$. LPS-rs was injected intrathecally using a Hamilton syringe with attached 30 gauge needle advanced at the lumbar spinal cord until a characteristic tail flick was noted.

The CCR2 antagonist, RS504393 (Santa Cruz Biotechnology), was dissolved in DMSO to administer $5 \mathrm{mg} / \mathrm{kg} /$ day via subcutaneous Alzet mini-pump (model 1002, $100 \mu \mathrm{l}$ volume, $0.25 \mu \mathrm{l} / \mathrm{hr}$, Alzet). Pumps delivering vehicle or RS504393 were placed subcutaneously in mice under isoflurane anesthesia through a $10 \mathrm{~mm}$ incision on the back which was then closed with surgical staples. Mini-pumps were removed after 2 weeks by reopening the prior incision under isoflurane anesthesia.

Tibial fracture complex regional pain syndrome (CRPS) model. Mice were anesthetized with isoflurane and underwent a closed right distal tibia fracture followed by casting. Briefly, the right hindlimb was wrapped in gauze, and a hemostat was used to make a closed fracture of the distal tibia. The hindlimb was then wrapped in casting tape (ScotchCast Plus) from the metatarsals of the hindpaw up to a spica formed around the abdomen to ensure that the cast did not slip off. The cast over the paw was applied only to the plantar surface with a window left open over the dorsum of the paw and ankle to prevent constriction when postfracture edema developed. Mice were inspected throughout the postoperative period of cast immobilization to ensure that the cast was properly positioned. At 3 weeks after fracture, mice were briefly anesthetized, and casts were removed with cast shears. For behavioral testing, mice were tested beginning one day after cast removal at week 3 until week 20 after fracture, as indicated in each section below. CRPS model generation and behavioral testing were conducting following well-established methods for evaluating mouse behavior in the tibial fracture-casting model of CRPS (Birklein et al., 2018; Guo et al., 2014).

Behavioral testing. To ensure rigor in our findings and avoid the contribution of experimenter sex to our behavioral data, experimenters were female or there was a female scientist's lab coat in the room during acclimation and testing (Sorge et al., 2014). In vivo behavioral testing was performed in a blinded fashion to the extent possible. All testing was conducted between 7:00 A.M. and 1:00 P.M. in an isolated, temperatureand light-controlled room. Mice were acclimated for 30-60 min in the testing environment within custom clear plastic cylinders (4 inch D) on a raised metal mesh platform (24 inch $\mathrm{H}$ ). Mice were randomized by simple selection from their home cage before testing, and placed in a cylinder; after testing, mouse identification numbers were recorded on the data sheet. No mice were excluded from the study.

Mechanical nociception assays. To evaluate mechanical reflexive hypersensitivity, we used a logarithmically increasing set of 8 von Frey filaments (Stoelting), ranging in gram force from 0.007 to $6.0 \mathrm{~g}$. These were applied perpendicular to the plantar hindpaw with sufficient force to cause a slight bending of the filament. A positive response was characterized as a rapid withdrawal of the paw away from the stimulus filament within 4 s. Using the up-down statistical method (Chaplan et al., 1994), the $50 \%$ withdrawal mechanical threshold scores were calculated for each mouse and then averaged across the experimental groups. Mechanical nociception testing was performed at baseline, then at weeks $3,5,7,9,12$, and 20 after fracture, as indicated in the figures.

Paw edema, unweighting, and temperature measurements. Hindpaw edema was determined by measuring the hindpaw dorsal-ventral thickness over the midpoint of the third metatarsal with a LIMAB laser measurement sensor (LIMAB) while the mouse was briefly anesthetized with isoflurane. Temperature and hindpaw thickness data were analyzed as the difference between the fracture side and the contralateral intact side and averaged across experimental groups. Paw edema was measured at week 3 after fracture.

An incapacitance device (IITC Life Science) was used to measure hindpaw unweighting. Mice were manually held in a vertical position over the apparatus with the hindpaws resting on separate metal scale plates, and the entire weight of the mouse was supported on the hindpaws. The duration of each measurement was $6 \mathrm{~s}$, and 6 consecutive measurements were taken at $60 \mathrm{~s}$ intervals. Six readings were averaged to calculate the bilateral hindpaw weight-bearing values. Unweighting was measured at baseline and then again at week 3 after fracture. 
The temperature of the hindpaw was measured using a fine-gauge thermocouple wire (Omega). Temperature testing was performed over the hindpaw dorsal skin between the first and second metatarsals (medial), the second and third metatarsals (central), and the fourth and fifth metatarsals (lateral). The measurements for each hindpaw were averaged for the mean paw temperature. Data were expressed as the average difference between the ipsilateral and contralateral hindpaw within an experimental group. Paw temperature was measured at week 3 after fracture.

Thermal nociception assays. To evaluate thermal-induced reflexive responses, we used the hotplate test (plate temperature was set to $52.5^{\circ}$ $\mathrm{C}$ ). Mice were placed on the plate and the latency (seconds) to the first appearance of a reflex response was recorded as a positive reflex withdrawal response. A maximal cutoff of $45 \mathrm{~s}$ was set to prevent tissue damage. Only one exposure to the hotplate was applied, to prevent behavioral sensitization that can result from multiple noxious exposures. Measurements were made at 5 weeks after fracture.

To evaluate affective-motivational (nonreflexive) responses to a sustained, inescapable noxious thermal stimulus, mice were placed on a $52.5^{\circ} \mathrm{C}$ hot plate for $45 \mathrm{~s}$ consistent with our previous use of this approach (Corder et al., 2017). A high-speed camera (on the side, level with the hot plate floor) was used to capture the movement, speed, velocity, and detailed reflexive and affective-motivational behaviors of the mice. We scored all behaviors, such as reflexive paw flinching (rapid flicking of the limb), paw attending (directed licking of the limb), paw guarding (intentional lift protection of the limb), and escape jumping for the duration of the trial. Videos were then scored blinded to treatment, for the total time spent engaging in reflexive (flinching) and nonreflexive (jumping, licking, guarding) behaviors, in addition to the latency to the first reflexive response, as previously described (Corder et al., 2017).

Immunohistochemistry. Mice (12-30 weeks) were transcardially perfused with $10 \%$ formalin in PBS. The spinal cord (lumbar cord L3-L5 segments) was dissected from the mice, cryoprotected in 30\% sucrose in PBS, and frozen in OCT (Fisher Healthcare Tissue-Plus). Spinal cord sections $(40 \mu \mathrm{m})$ were prepared using a cryostat (Leica Biosystems) and incubated in blocking solution (5\% normal donkey serum and $0.3 \%$ Triton X-100 in PBS) for $1 \mathrm{~h}$ at room temperature followed by incubation with primary antibodies at $4^{\circ} \mathrm{C}$ overnight. The following primary antibodies were used: rat anti-CD11b (Bio-Rad, \#MCA711G, 1:500), mouse anti-GFAP (Sigma Millipore, \#G3893, 1:400), rabbit anti-Iba1 (Wako, \#019-19741, 1:500), and goat anti-GFP (Abcam, \#ab6673, $1: 1000)$. After extensive wash with $1 \%$ normal donkey serum and $0.3 \%$ Triton X-100 in PBS, sections were incubated with appropriate secondary antibody conjugated to AlexaFluor for $2 \mathrm{~h}$ at room temperature. Sections were counterstained with DAPI and mounted on slides using Fluoromount aqueous mounting medium (Thermo Fisher Scientific, $\# 00-4959-52$ ). Images were collected with a Keyence BZ-X800 fluorescent microscope (Keyence) using the sectioning module to remove nonfocused light using the $20 \times$ or $60 \times$ objective magnification. Each image was taken with identical exposure with $0.4-\mu \mathrm{m}$-step $z$ stacks of 19 slices. We collected 3-6 dorsal horn images per mouse to evaluate the relative cellular expression of Iba1 and GFAP in the spinal cord dorsal horn. Images were analyzed using ImageJ/FIJI (Schindelin et al., 2012) by outlining the dorsal horn and setting the threshold to detect only the area of staining and not the background. An identical threshold was then set for all the images and expressed as percent staining Iba1 or GFAP-positive over the entire area of the dorsal horn. The results were then normalized to $\mathrm{TLR}^{+/+}$controls that also underwent fracture (the same TLR4 ${ }^{+/+}$ controls were used for early and late TLR4 CKO IHC), and final data are shown as $\% \mathrm{Ibal}^{+} /$control or $\% \mathrm{GFAP}^{+} /$control. In addition, we evaluated the expression of Ibal and GFAP in individual Rexed laminae I-IV of the spinal cord dorsal horn by setting laminar boundaries in NeuN-stained spinal cord based on neuronal density. These laminar boundaries were then used to set each image for quantification using ImageJ/FIJI by outlining each respective lamina and setting thresholds identical to the total spinal cord dorsal horn.

$q R T-P C R$. Tissue was collected and placed in TRIzol Reagent (Invitrogen, \#15596018). Once all samples were collected, the tissue was homogenized and placed in $-20^{\circ} \mathrm{C}$ until the RNA isolation step. Sorted cells were collected directly into Lysis Buffer RLT (QIAGEN, \#74034). Once all samples were collected, the cells were stored at $-80^{\circ} \mathrm{C}$ until the RNA isolation. RNA was isolated per the manufacturer's instructions. The concentration and purity of RNA samples were determined using NanoDrop 2000 (Thermo Fisher Scientific) for tissue samples and using the RNA pico assay on the Agilent 2100 Bioanalyzer (Agilent Technologies) for sorted cells. RNA was reverse transcribed with $\mathrm{RT}^{2}$ First Strand Kit per manufacturer's instructions (QIAGEN, \#330404).

qPCR analysis was performed with PowerUp SYBR Green Master Mix (Thermo Fisher Scientific, \#A25741) and $\mathrm{RT}^{2}$ qPCR Primer Assays for Itgam (QIAGEN, \#PPM03671F), Gapdh (QIAGEN, \#PPM02946E), and Tlr4 (QIAGEN, \#PPM04207F) on an Applied Biosystems 7900HT or on an Applied Biosystems StepOnePlus. Appropriate no reverse-transcriptase and no template controls were used for each 96- or 384-well PCR reaction. The cycle conditions were as follows: $50^{\circ} \mathrm{C}$ for $2 \mathrm{~min}, 95^{\circ} \mathrm{C}$ for $2 \mathrm{~min}$, then 40 cycles of $15 \mathrm{~s}$ at $95^{\circ} \mathrm{C}, 1 \mathrm{~min}$ at $60^{\circ} \mathrm{C}$. Dissociation analysis was performed at the end of each run to ensure specificity. Relative quantification of gene expression was performed via $2^{-\Delta \Delta C(T)}$ method (Livak and Schmittgen, 2001).

Brain and spinal cord cell dissociation. Mice were anesthetized with $120 \mathrm{mg} / \mathrm{kg}$ ketamine and $5 \mathrm{mg} / \mathrm{kg}$ xylazine and perfused with $15 \mathrm{ml}$ icecold Medium A (50 $\mathrm{ml} 1 \times$ HBSS without $\mathrm{Ca}^{2+}$ or $\mathrm{Mg}^{2+}$ [Invitrogen, \#14185052], $750 \mu \mathrm{l} 1 \mathrm{~m}$ HEPES [Invitrogen, \#15630080], $556 \mu \mathrm{l} 45 \%$ glucose [Sigma Millipore, \#G8769]). Brains or spinal cords were placed in 2 $\mathrm{ml}$ Medium A $+80 \mu \mathrm{l}$ DNase I (12,400 units/ml, Worthington, \#LS002007) until all samples were dissected. Samples were dounce-homogenized, passed through a $100 \mu \mathrm{m}$ strainer, washed with $5 \mathrm{ml}$ Medium A, and spun down by centrifugation at $340 \times g$ for $7 \mathrm{~min}$ at $4^{\circ}$ C. Supernatant was suctioned, and pellets were resuspended in $6 \mathrm{ml} 25 \%$ Standard Isotonic Percoll (GE Healthcare, \#17-5445-02 with 10\% 10× PBS) in Medium A. The suspension underwent centrifugation for $20 \mathrm{~min}$ at $950 \times \mathrm{g}$ at $4^{\circ} \mathrm{C}$ to remove myelin. Supernatant was discarded; then the pellet was washed with $5 \mathrm{ml}$ Medium $\mathrm{A}$ and spun down by centrifugation at $340 \times g$ for $7 \mathrm{~min}$ at $4^{\circ} \mathrm{C}$. Cells were resuspended in FACS buffer (5 mm EDTA in 1\% BSA in $1 \times$ PBS).

Spleen cell dissociation. Spleens were collected at the same time as brain or spinal cord collection and placed in $5 \mathrm{ml} 1 \times$ PBS without $\mathrm{Ca}^{2+}$ or $\mathrm{Mg}^{2+}$ until all tissue was collected. Spleens were homogenized by placing the tissue between two slides until a homogeneous solution was obtained, then immediately passed through a $100 \mu \mathrm{m}$ strainer and washed with $5 \mathrm{ml} 1 \times$ PBS. Samples were spun down by centrifugation at $340 \times g$ for $7 \mathrm{~min}$ at $4^{\circ} \mathrm{C}$. Cells were resuspended in $2 \mathrm{ml}$ ACK lysing buffer (Invitrogen, \#A1049201) and placed in a rotator for $5 \mathrm{~min}$ at room temperature; then cells were spun down at $300 \times g$ for $5 \mathrm{~min}$ at $4^{\circ} \mathrm{C}$. ACK lysing buffer was suctioned off and resuspended in $5 \mathrm{ml} 1 \times \mathrm{PBS}$, then spun down by centrifugation at $340 \times g$ for $7 \mathrm{~min}$ at $4^{\circ} \mathrm{C}$. PBS was suctioned off and cells were resuspended in FACS buffer.

Flow cytometry and FACS. The following antibodies were used for flow cytometry: e450-conjugated anti-CD3 (eBioscience, \#48-0031-82), e450-conjugated anti-CD19 (eBioscience, \#48-0193-82), PE-Cy7-conjugated anti-CD45 (Biolegend, \#103114), and APC-conjugated antiCD11b (Biolegend, \#101212). Dead cells were stained with 1:1000 SYTOX Blue (Invitrogen, \#S34857). Cell suspension was then spun down at $300 \times g$ for $5 \mathrm{~min}$ at $4^{\circ} \mathrm{C}$. The FACS buffer supernatant was suctioned off, and cells were resuspended in fresh FACS buffer. Before staining, all samples were preblocked with 1:100 anti-CD16/CD32 (BD Pharmingen, \#553142) for $5 \mathrm{~min}$ at room temperature. Afterward, all antibodies were added to the samples at 1:100 along with 1:1000 SYTOX Blue and placed on ice for $30 \mathrm{~min}$. Samples were spun down for $5 \mathrm{~min}$ at $400 \times g$ at $4^{\circ} \mathrm{C}$, suctioned, and replaced with fresh FACS buffer. Suspension was then spun down for $5 \mathrm{~min}$ at $400 \times g$ at $4^{\circ} \mathrm{C}$. Supernatant was suctioned off; then cells were again resuspended in FACS buffer and passed through $35 \mu \mathrm{m}$ filter into polystyrene tubes. Samples were analyzed by flow cytometry in an LSRII (BD Biosciences) or analyzed and sorted using a BD Aria II (BD Biosciences). Flow cytometry analysis was done using FlowJo version 10.6.2. Single cells were gated for live (SYTOX blue negative), $\mathrm{CD}_{1}{ }^{-} \mathrm{CD}^{-} \mathrm{CD}^{-} 5^{\text {mid }}$ then $\mathrm{CD}_{11 \mathrm{~b}^{+}}$and $\mathrm{Cx} 3 \mathrm{CR} 1-\mathrm{YFP}^{+}$to isolate microglia from spinal cord or 
Table 1. Student's $t$ test and one- or two-way ANOVA with their respective post-hoc comparisons for each figure

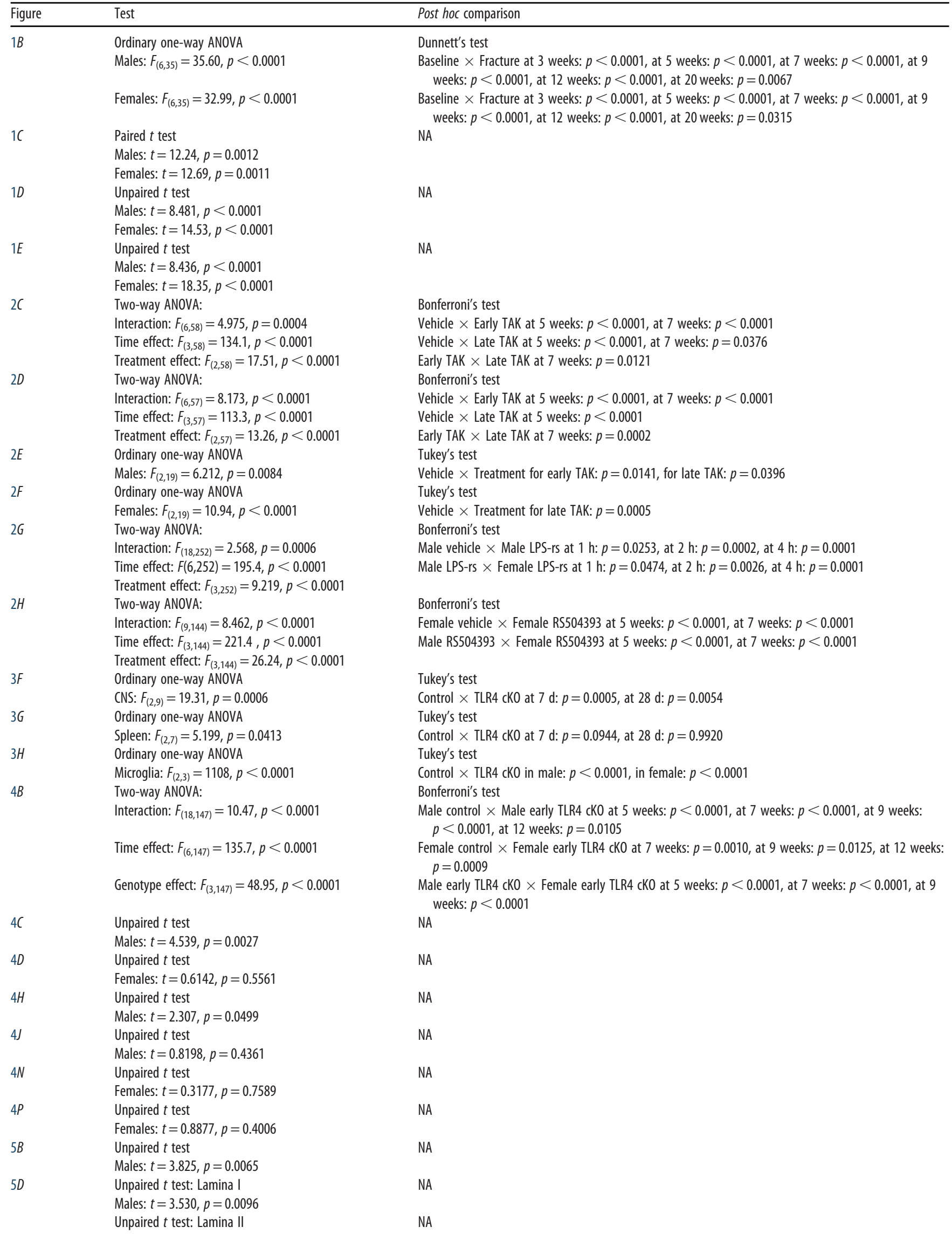


Table 1. Continued

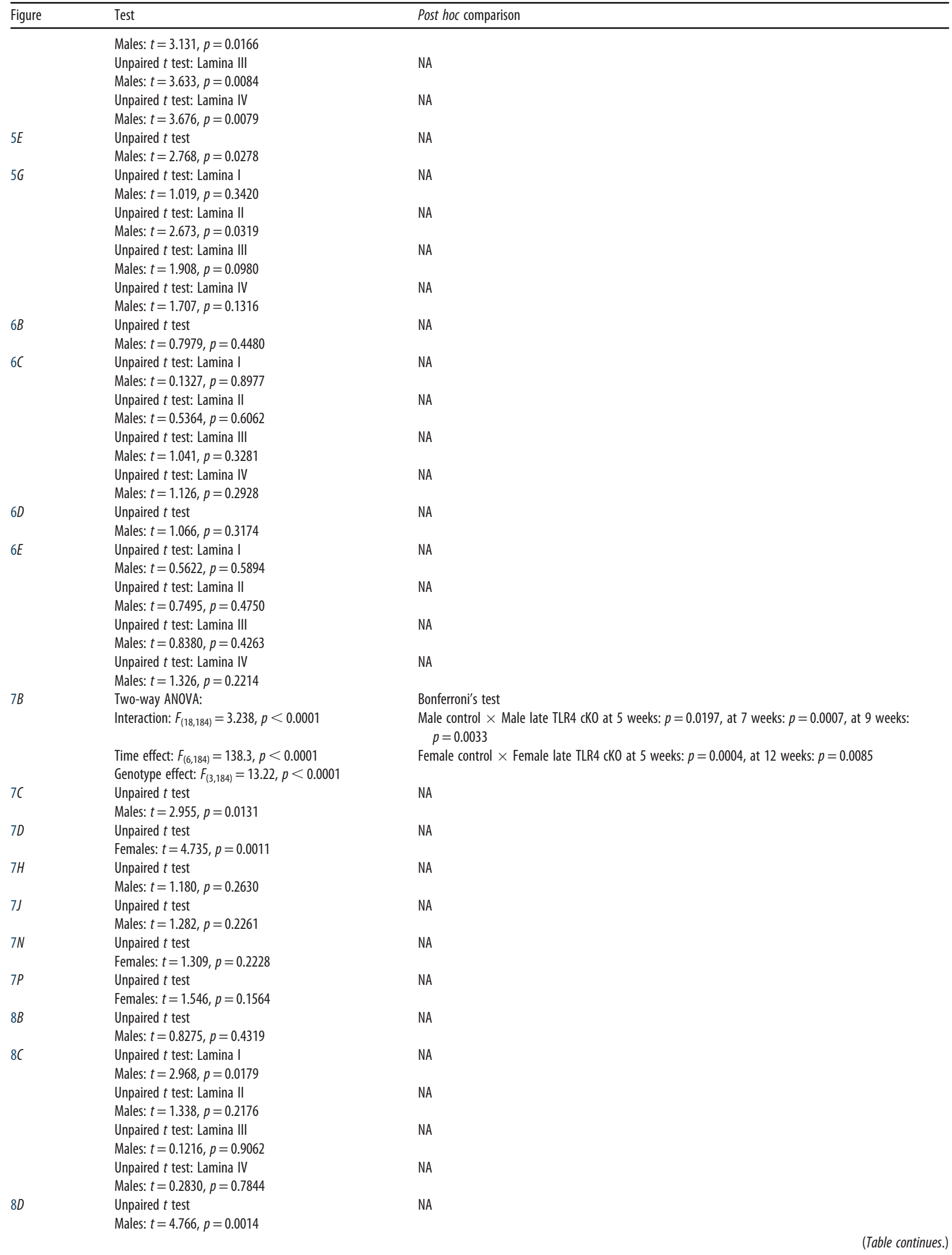


Table 1. Continued

\begin{tabular}{|c|c|c|}
\hline Figure & Test & Post hoc comparison \\
\hline \multirow[t]{6}{*}{$8 E$} & Unpaired $t$ test: Lamina I & NA \\
\hline & Males: $t=1.657, p=0.1360$ & \\
\hline & Unpaired $t$ test: Lamina II & NA \\
\hline & Unpaired $t$ test: Lamina III & NA \\
\hline & Males: $t=4.453, p=0.0021$ & \\
\hline & Unpaired $t$ test: Lamina IV & NA \\
\hline $9 B$ & Males: $t=0.5207, p=0.6152$ & \\
\hline \multirow[t]{5}{*}{$9 C$} & Unpaired $t$ test: Lamina I & NA \\
\hline & Males: $t=2.753, p=0.0224$ & \\
\hline & Unpaired $t$ test: Lamina II & NA \\
\hline & Males: $t=0.7424, p=0.4768$ & \\
\hline & Unpaired $t$ test: Lamina III & NA \\
\hline $9 D$ & Males: $t=4.058, p=0.0028$ & \\
\hline \multirow[t]{8}{*}{$9 E$} & Unpaired $t$ test: Lamina I & NA \\
\hline & Males: $t=5.975, p=0.0002$ & \\
\hline & Unpaired $t$ test: Lamina II & NA \\
\hline & Males: $t=4.118, p=0.0026$ & \\
\hline & Unpaired $t$ test: Lamina III & NA \\
\hline & Males: $t=2.921, p=0.0170$ & \\
\hline & Unpaired $t$ test: Lamina IV & NA \\
\hline & Males: $t=1.676, p=0.1281$ & \\
\hline
\end{tabular}

brain. For Cx3CR1-Cre $e^{E R T 2-E Y F P}$; R26-tdTomato mice, cells were additionally gated on tdTomato.

Experimental design and statistical analysis. Cohort sizes were determined based on historical data from our laboratory using a power analysis to provide $>80 \%$ power to discover $25 \%$ differences with $p<0.05$ between groups to require a minimum of 5 animals per group for all behavioral outcomes, 2 animals per group for PCR analyses, and 3 animals per group for IHC analyses. All experiments were randomized by cage and performed by a blinded researcher. Researchers remained blinded throughout histologic, biochemical, and behavioral assessments. Groups were unblinded at the end of each experiment before statistical analysis. Data are expressed as the mean \pm SEM. Statistical analysis was performed using GraphPad Prism version 8.4.1 (GraphPad Software). Data were analyzed using a Student's $t$ tests, or ordinary one-way with Dunnett's or Tukey's post hoc test, or two-way analysis of variance with a Bonferroni post hoc test, as indicated in the main text or figure legends, as appropriate, with complete statistical analyses detailed in Table 1 . The $n$ value for each individual experiment is listed in the figure legends.

Data availability. All data supporting the findings of this study are available on reasonable request.

\section{Results}

Males and females exhibit spinal cord dorsal horn microglial activation after injury in the tibial fracture model of CRPS

We first sought to establish the time course of glial activation in the spinal cord after tibial fracture, a well-characterized mouse model of the pain condition CRPS (Birklein et al., 2018). Mice underwent unilateral closed tibial fracture and cast placement for 3 weeks, after which the cast was removed, and behavioral testing was conducted (Fig. 1A). Both males and females developed persistent allodynia as evidenced by a decrease in mechanical threshold from the time of cast removal at 3 weeks, through 12 weeks after after injury (Fig. 1B). As previously demonstrated (Gallagher et al., 2013; Cropper et al., 2019), mice developed
CRPS-like signs, including erythema, unweighting, increased temperature, and edema of the injured paw (Fig. 1C-E). Spinal cord dorsal horn microglial activation, as evidenced by increased CD11b expression and morphologic change, was noted as early as 1 week after injury, peaked at 3 weeks after injury, and persisted until at least 7 weeks after injury in males (Fig. 1F; data not shown) and females (Fig. 1G; data not shown). In addition, astrocytic activation, as evidenced by increased GFAP expression and morphologic change, was noted with slight delay, starting at 3 weeks after injury and persisting until at least 7 weeks after injury in males (Fig. 1F; data not shown) and females (Fig. 1G; data not shown).

\section{A systemically available TLR4 antagonist improves recovery} trajectory differentially in male and female mice

Given the clear time course of microglial activation in our tibial fracture model, we next investigated how inhibition of TLR4, a receptor that triggers myeloid cell activation (Grace et al., 2014a), may alter nociceptive sensitization and CRPS signs. We first used a pharmacologic approach with the systemically available TLR4 antagonist, TAK242 (resatorvid). We selected a dose of $3 \mathrm{mg} / \mathrm{kg}$ (i.p.) daily based on previous work that demonstrated sustained plasma and CNS levels for $24 \mathrm{~h}$ after dosing (Hua et al., 2015). TAK 242 was dosed for $14 \mathrm{~d}$, either starting at the time of injury ("early," Fig. 2A) or at the time of cast removal at 3 weeks ("late," Fig. 2B). Early treatment with TAK242 improved weight bearing and decreased edema of the injured paw only in males, whereas late treatment with TAK242 improved temperature in males and females without any effect on other CRPS signs (data not shown). Early treatment with TAK242 did not alter acute allodynia in males or females; at the time of cast removal (3 weeks), all mice exhibited robust mechanical allodynia that 
A

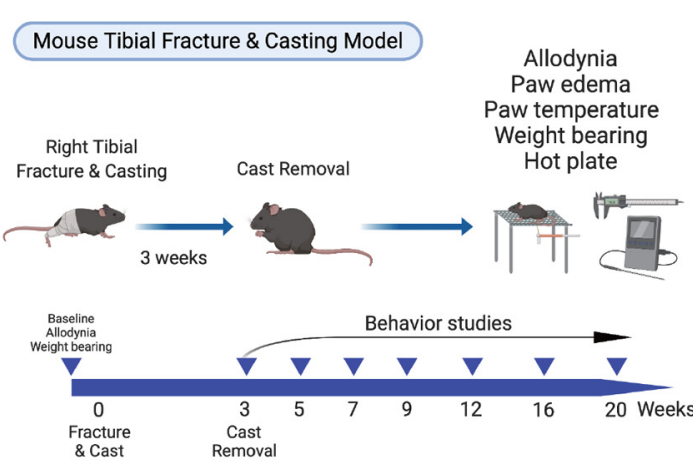

C

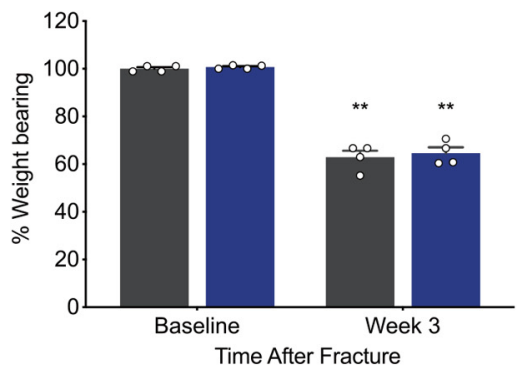

F Male

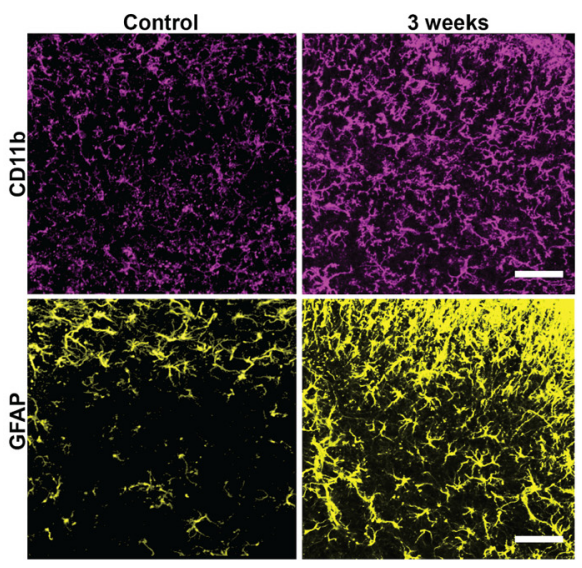

B

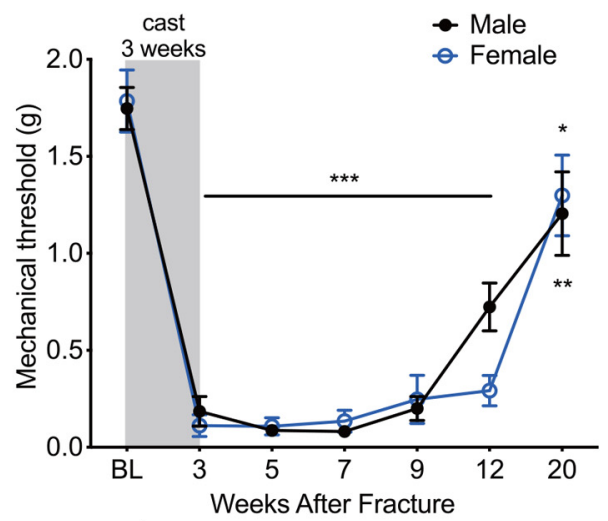

E

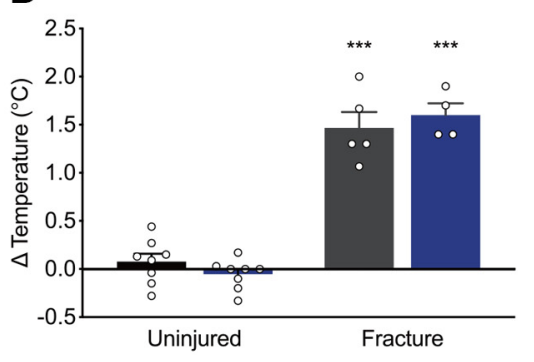

Male

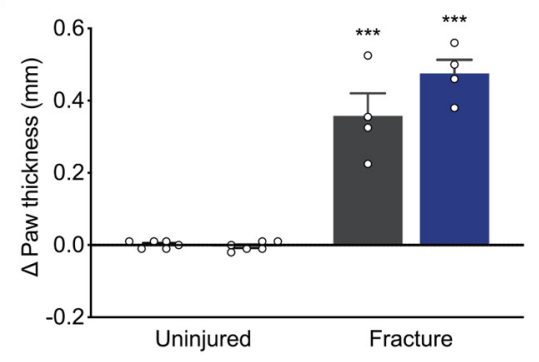

Female

\section{G Female}

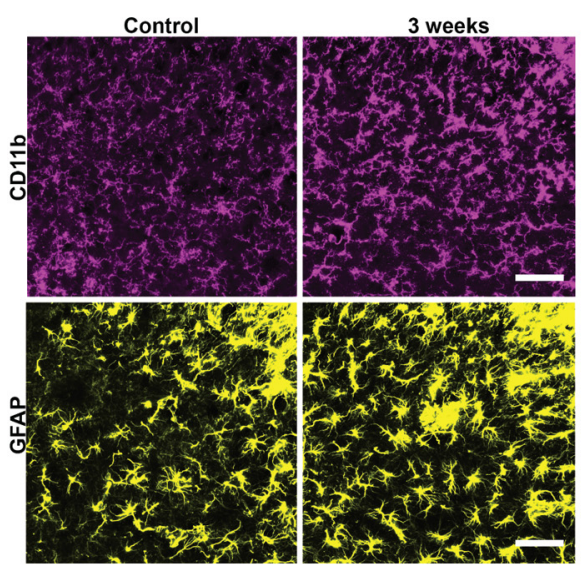

Figure 1. Tibial fracture/casting model reproduces classic signs of CRPS, including long-lasting allodynia. $\boldsymbol{A}$, General experimental scheme for the mouse tibial fracture and casting model. $\boldsymbol{B}$, Profound and long-lasting mechanical allodynia after cast removal lasts through 20 weeks after injury in males and females. $n=6$ or 7 mice per group. $* p<0.05 ; * * p<0.01$; $* * * p<0.001$ versus sex-matched baseline threshold by one-way ANOVA with Dunnett's post-test. $C$, After cast removal at 3 weeks, male and female mice placed less weight on the injured limb compared with baseline. $n=4$ per sex. $* * p<0.01$ versus sex-matched baseline value by paired $t$ test. $\boldsymbol{D}$, The ipsilateral/injured paw exhibited an increase in temperature compared with the contralateral/uninjured paw in both males and females at week 3 after fracture. $n=5-8$ males; 4-8 females. $* * * p<0.001$ versus uninjured sex-matched control by unpaired $t$ test. $\boldsymbol{E}$, Injured paws were edematous as indicated by an increase in paw thickness compared with the uninjured paw in both sexes at 3 weeks after fracture. $n=4-6$ per sex. $* * * p<0.001$ versus uninjured sex-matched control by unpaired $t$ test. Immunohistochemistry in the lumbar spinal cord dorsal horn revealed increases in microglial (CD11b/magenta) and astrocytic (GFAP/yellow) activation at 3 weeks after injury in males $(\boldsymbol{F})$ and females $(\boldsymbol{G})$. Images taken at $20 \times$ with an additional $2.5 \times$ zoom after capture. Scale bar, $25 \mu \mathrm{m}$. Data are mean \pm SEM; all data points are shown in $\mathbf{C}-\boldsymbol{E}$.

was not significantly different from vehicle-treated controls (Fig. 2C,D). The trajectory of allodynia in males was, however, significantly altered by both early or late TAK242, which resulted in persistent reversal of mechanical allodynia out to 7 weeks after injury (Fig. 2C). In females, both early and late TAK242 reversed injury-induced allodynia at 5 weeks, but only early TAK2 42 had a persistent effect out to 7 weeks after injury (Fig. 2D). Similarly, early TAK242 had a significant effect on thermal latency only in males (Fig. 2E), while late TAK242 significantly increased thermal latency in both males (Fig. 2E) and females (Fig. 2F). We further investigated the effect of TAK242 on reflex and nonreflexive behaviors to thermal stimulus by scoring flinching, guarding, jumping, and licking over $45 \mathrm{~s}$. Early TAK242 did not affect the cumulative duration of behaviors or cumulative summation of flinches in males or females (data not shown). Late TAK242 did not affect the cumulative duration of behaviors or cumulative summation of flinches in males; however, we did identify a significant decrease in the cumulative summation of flinches in female mice (data not shown). 
A

Early TAK242 dosing 3mg/kg i.p.

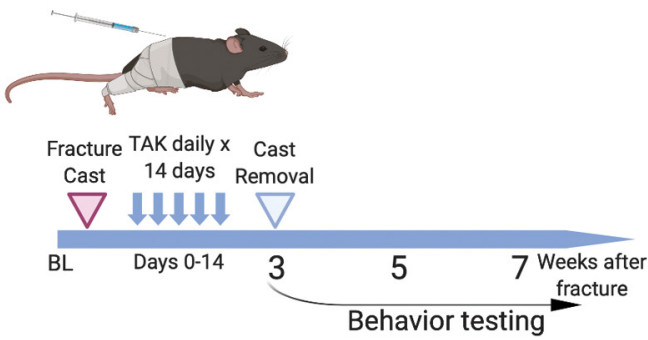

C

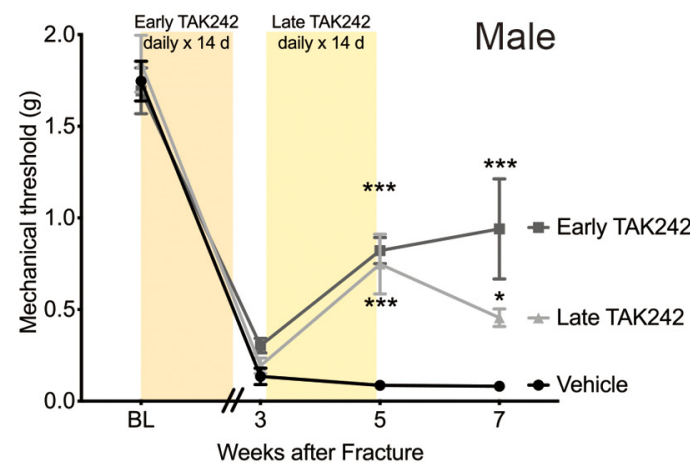

E

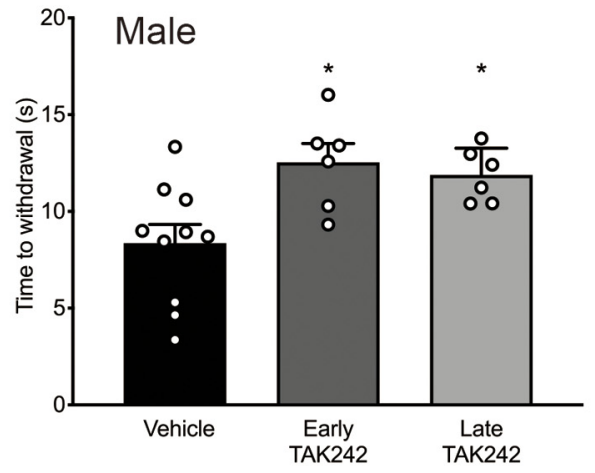

G

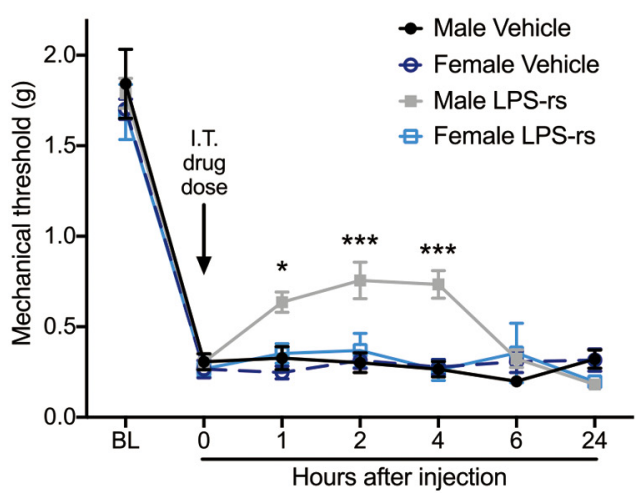

B
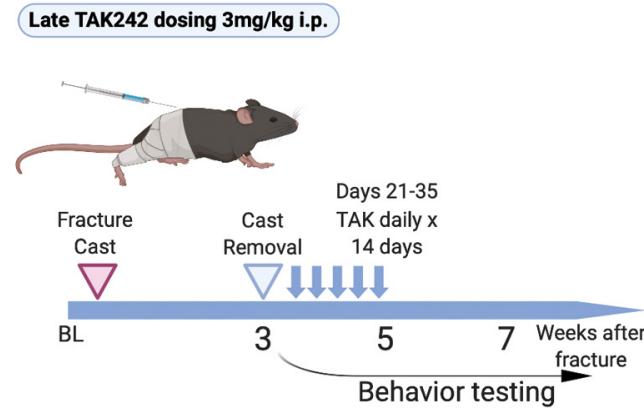

D

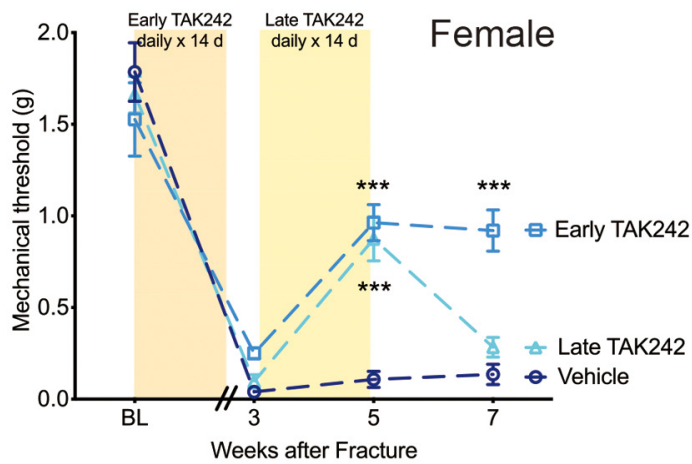

F

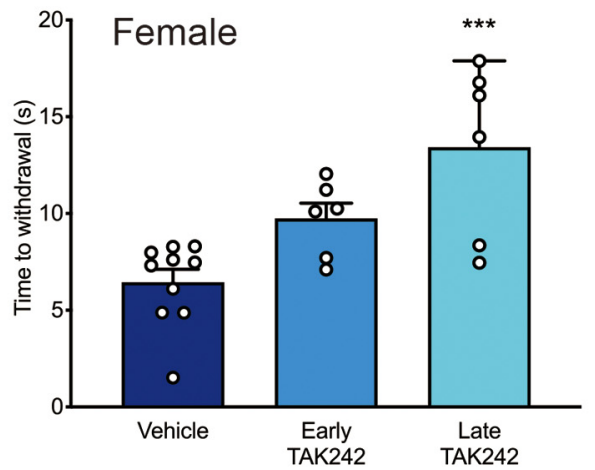

H

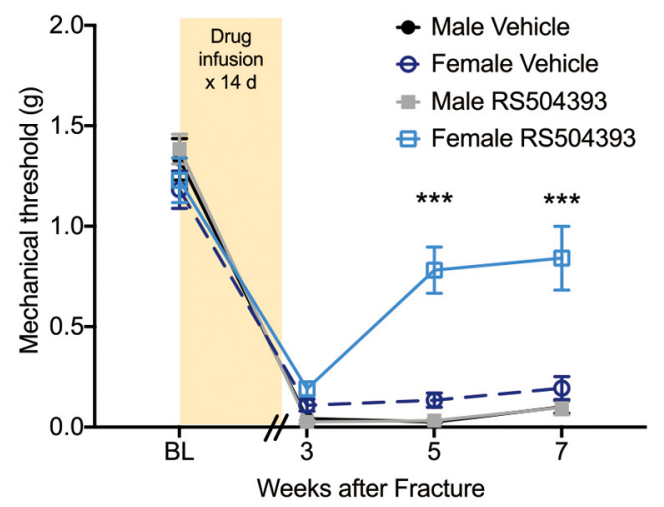

Figure 2. Early treatment with the systemic TLR4 antagonist, TAK242, results in sustained improvement in allodynia in both sexes. Experimental scheme for treatment with the systemic TLR4 antagonist, TAK242, either at the time of injury (early, $\boldsymbol{A}$ ) or at the time of cast removal (late, $\boldsymbol{B}$ ). Mechanical threshold of the injured paw decreases after cast removal at 3 weeks but rapidly reverses trajectory, an effect that was sustained in males $(\boldsymbol{C})$ and females $(\boldsymbol{D})$ with early TAK242 dosing and sustained only in males with late TAK242 dosing. $n=3-6$ per group per time point. $* p<0.05 ; * * * p<0.001$ versus sex-matched vehicle control by two-way ANOVA with Bonferroni's post-test. $\boldsymbol{E}$, Both early and late treatment with TAK242 increases hot plate latency at 5 weeks after injury in males. $n=6-10$ mice per group. $* p<0.05$ versus sex-matched vehicle control by one-way ANOVA with Tukey's post-test. $\boldsymbol{F}$, Late treatment with TAK242 increases hot plate latency at 5 weeks after injury in females. $n=6-10$ mice per group. $* * * p<0.001$ versus sex-matched vehicle control by one-way ANOVA with Tukey's post-test. G, Late intrathecal injection of the TLR4 antagonist, LPS-rs, improves male, but not female, allodynia at 3 weeks after injury. $n=10$ mice per group. $* p<0.05 ; * * * p<0.001$ versus male vehicle control by two-way ANOVA with Bonferroni's post-test. $\boldsymbol{H}$, Early, continuous infusion of the peripheral CCR2 antagonist, RS504393, improves persistent, but not acute, allodynia in females at 5 and 7 weeks after injury. $n=10$ mice per group. $* * * p<0.001$ versus female vehicle control by two-way ANOVA with Bonferroni's post-test. 
A

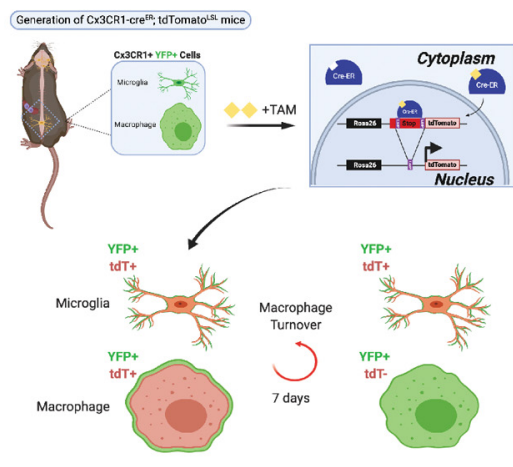

B

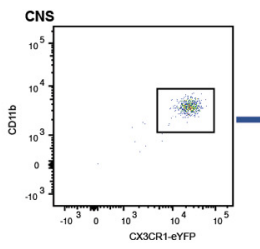

C
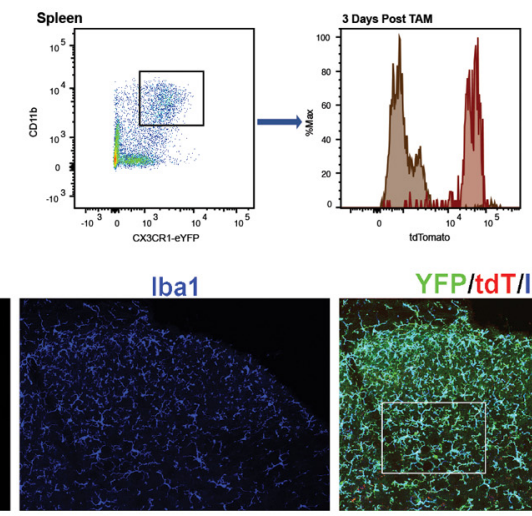
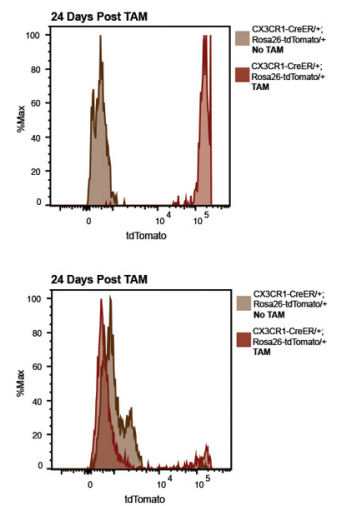

D
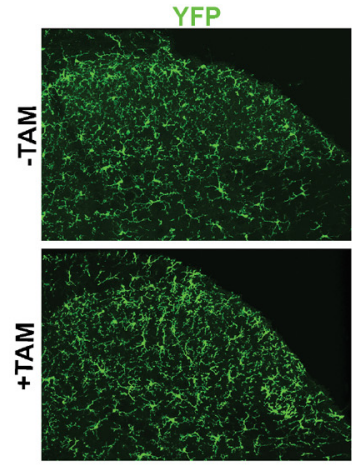

E

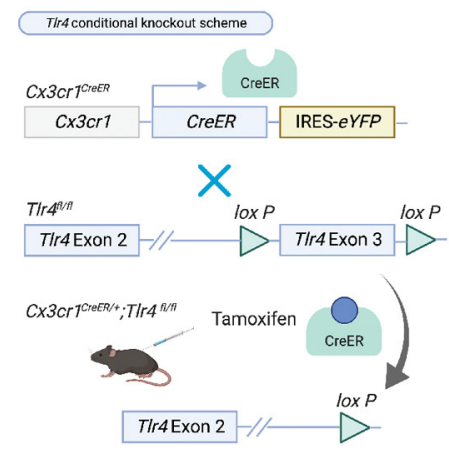

tdT
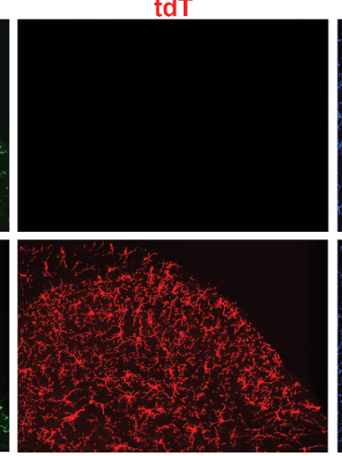

$\mathbf{F}$

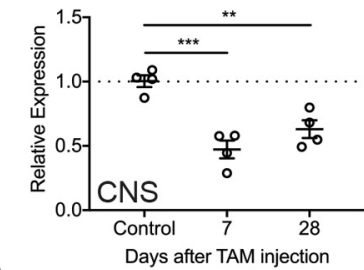

G

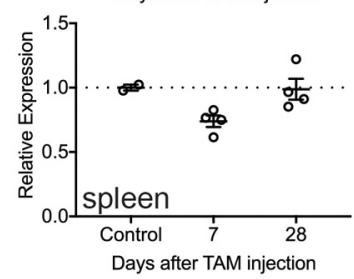

lba1

YFP/tdT/lba1
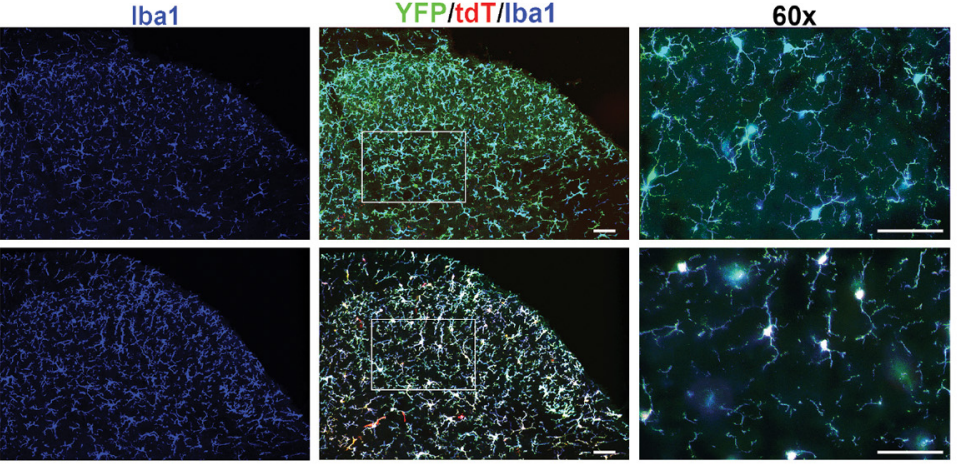

H
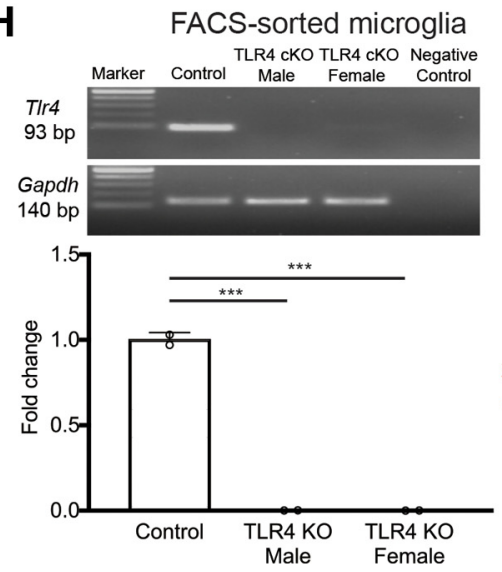

Figure 3. TLR4 CKO is tamoxifen-dependent and microglia-specific. A, CX3CR1-Cre $e^{\text {ERT2-eYFP }}$; tdTomato ${ }^{L S L}$ mice were generated in which all Cx3CR1 ${ }^{+}$cells express the tdTomato transgene after tamoxifen-dependent Cre recombination. Differential turnover rates of central microglia versus peripheral macrophages result in persistent tdTomato expression in microglia. $\boldsymbol{B}$, Flow cytometry

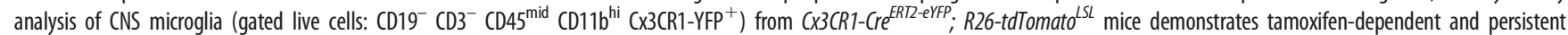

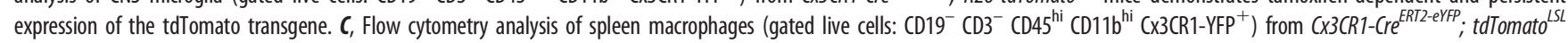
mice demonstrates tamoxifen-dependent and transient expression of the tdTomato transgene with expression of tdTomato at 3, but not $24, \mathrm{~d}$ after tamoxifen treatment. $\boldsymbol{D}$, CX3CR1-Cre ERT2-eYFP ; R26-tdTomato mice were generated, and sections from lumbar spinal cord demonstrate tdTomato expression that is tamoxifen-dependent and overlaps fully with CX3CR1-

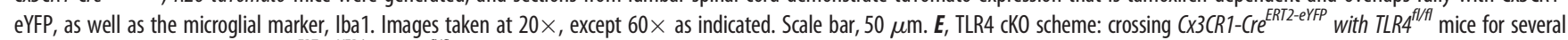
generations resulted in $C X 3 C R T-C r e^{E R T 2-e Y F P /+} ;$;LR4 ${ }^{f / f}$ mice in which tamoxifen administration resulted in KO of TLR4 exon 3. F, TIr4 mRNA expression was evaluated in whole brain by realtime RT-PCR and demonstrates persistent knockdown in CNS out to $28 \mathrm{~d}$. There remains some TIr4 mRNA in whole CNS tissue, likely ascribed to neuronal or astrocytic expression. $n=2-4$ mice per group. $* * p<0.01 ; * * * p<0.001$ versus controls by one-way ANOVA with Tukey's post-test. G, TIr 4 mRNA expression was evaluated in spleen by real-time RT-PCR and demonstrates no significant decrease in tlr4 mRNA in spleen. $\boldsymbol{H}$, Microglia were isolated from adult brain 3 weeks after tamoxifen using FACS (gated live cells: $\mathrm{CD}_{19} \mathrm{CD}^{-} \mathrm{CD}^{-} 5^{\mathrm{mid}} \mathrm{CD} 11 \mathrm{~b}^{\mathrm{hi}} \mathrm{C} \times 3 \mathrm{CR} 1^{+}$) followed by real-time RT-PCR, which showed normal expression of trr4 mRNA in the control and undetectable levels in the male and female TLR4 cKO samples. RNA gels shown on top with Gapdh as the housekeeping gene control and sample with no reverse transcription as the negative control. Fold change from control shown in graph with $n=2$ mice per group. $* * * p<0.001$ versus control by one-way ANOVA with Tukey's post-test.

Given that TAK242 crosses the blood-brain barrier (Hua et al., 2015), in a separate set of experiments, we determined whether intrathecal dosing of the TLR4 antagonist, LPS-rs, would have a sex-specific effect when given at the "late" time point. Consistent with previous work in the spared nerve injury model of neuropathic pain (Sorge et al., 2011), we found that $10 \mu \mathrm{g}$ of intrathecal LPS-rs resulted in transient reversal of mechanical allodynia only in males, with an effect that lasted $4 \mathrm{~h}$ (Fig. 2G). In order to clarify the contribution of peripheral versus central myeloid lineage cells to allodynia after injury, we next used the peripheral CCR2 antagonist, RS504393, to inhibit peripheral macrophages (Raghu et al., 2017). A $14 \mathrm{~d}$ infusion of RS504393 by subcutaneous osmotic mini-pump had no effect on acute allodynia; at the time of cast removal (3 weeks), all mice 
A
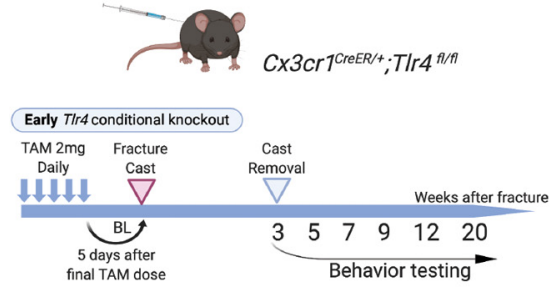

E

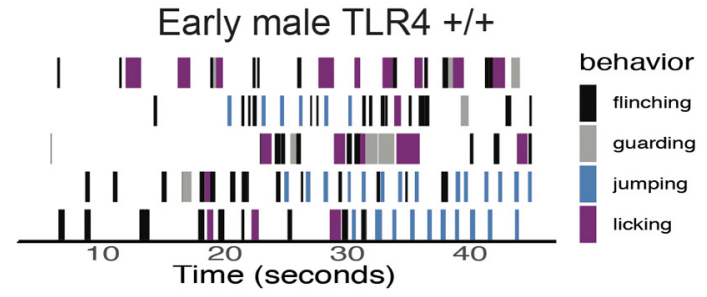

G

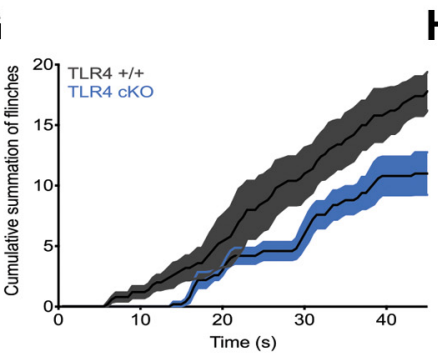

H

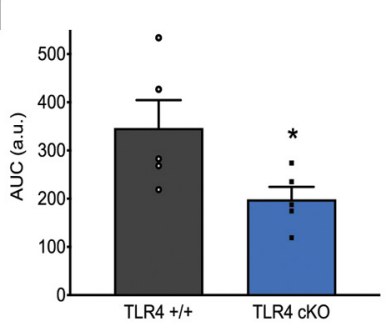

K

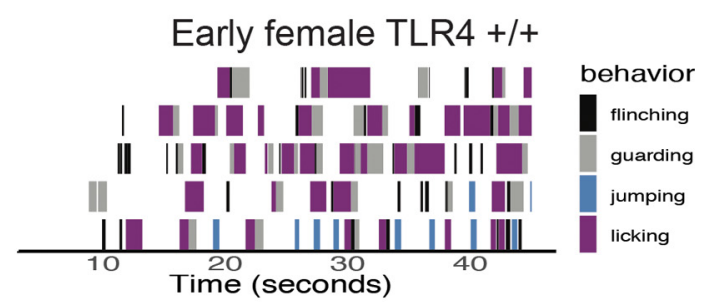

M
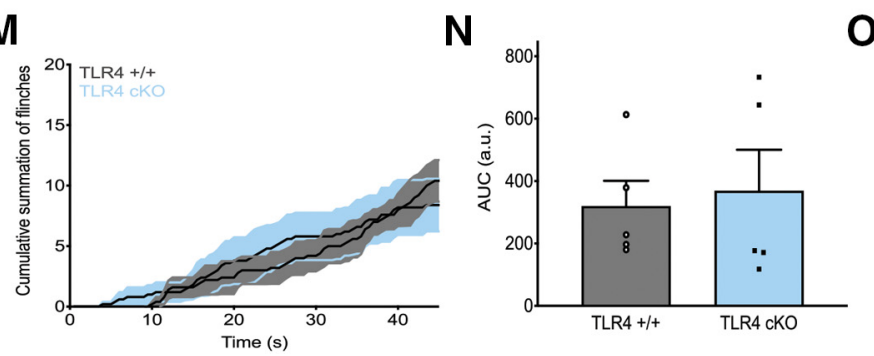

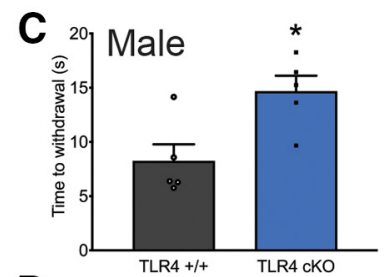

D

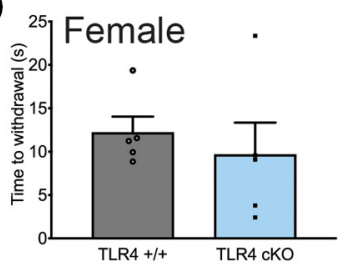

Weeks after Fracture
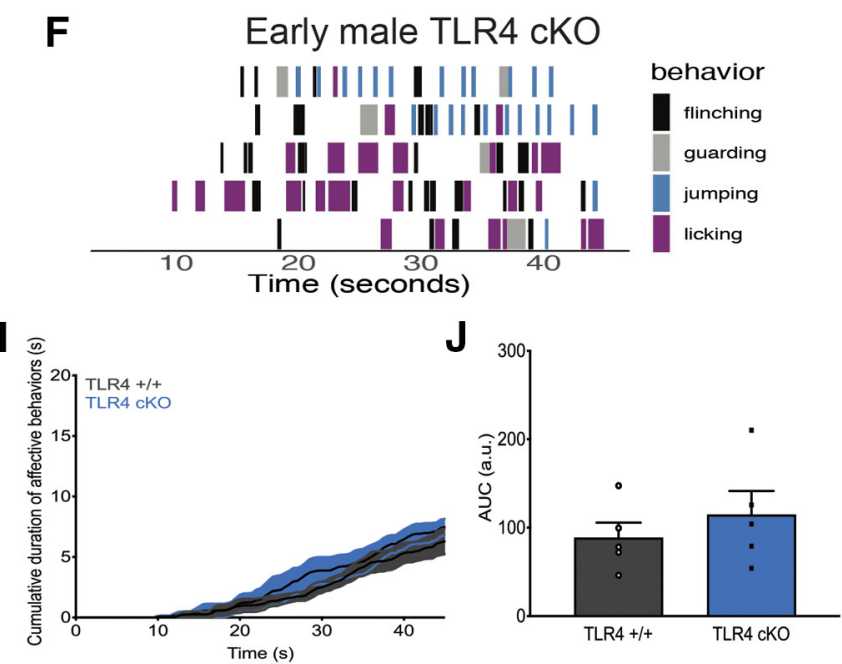

L Early female TLR4 cKO
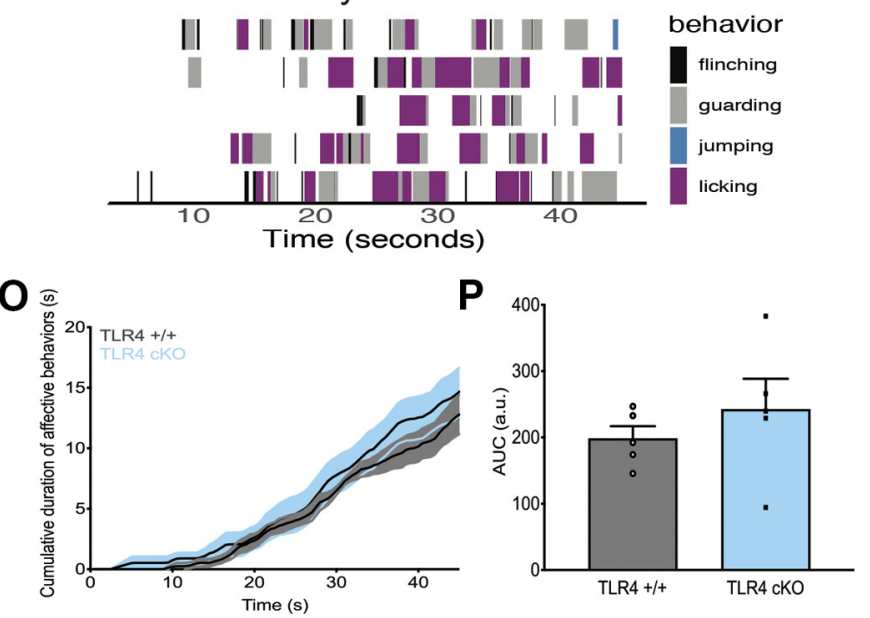

Figure 4. Early cKO of microglial TLR4 improves allodynia and thermal hyperalgesia in a time- and sex-specific manner without affecting acute sensitization. $\boldsymbol{A}$, Experimental scheme for early TLR4 KO in microglia. $\boldsymbol{B}$, Early male TLR4 CKO results in a complete and sustained reversal of existing allodynia, whereas early female TLR4 cKO significantly improves allodynia but less strikingly. $n=5$-10 per group. $* p<0.05 ; * * p<0.01 ; * * * p<0.001$ versus sex-matched control by two-way ANOVA with Bonferroni's post-test. C, Early male TLR4 cK0 resulted in a significantly increased latency to withdrawal on a $52.5^{\circ} \mathrm{C}$ hot plate; however, this effect was not significant in the early females TLR4 CKO group (D). Mice were placed on an inescapable $52.5^{\circ} \mathrm{C}$ hot plate, and latency to first reflexive response was measured at 5 weeks after fracture. $n=5$ per group. $* p<0.05$ versus sex-matched control. All behavioral responses [flinching (black), guarding (gray), jumping (blue), or licking (purple)] were scored for $45 \mathrm{~s}$ on a $52.5^{\circ} \mathrm{C}$ hot plate for early male TLR4 ${ }^{+/+}(\boldsymbol{E})$ and TLR4 cK0 mice $(\boldsymbol{F})$. Each row represents 1 mouse. $n=5$ per group. $\boldsymbol{G}$, Cumulative summation of flinches over $45 \mathrm{~s}$ for early male TLR4 ${ }^{+/+}$(dark gray) and TLR4 CKO mice (dark blue). $\boldsymbol{H}$, Area under the curve (AUC) for summation of flinches demonstrates significant decrease in number of flinches in early male TLR4 cKO. $n=5$ per group. $* p<0.05$ versus sex-matched control by unpaired $t$ test. $I$, Cumulative duration of nonreflexive behaviors (guarding, jumping, licking) over $45 \mathrm{~s}$ for TLR4 ${ }^{+/+}$(dark gray) or early TLR4 CKO (dark blue) male mice. J, AUC for duration of nonreflexive behaviors demonstrates no change with early TLR4 cKO in males. $n=5$ per group. All behavioral responses were scored for $45 \mathrm{~s}$ on a $52.5^{\circ} \mathrm{C}$ hot plate for early female TLR4 $4^{+/+}(\boldsymbol{K})$ and TLR4 cKO mice $(\boldsymbol{L})$. Each row represents 1 mouse. $n=5$ per group. $\boldsymbol{M}$, Cumulative summation of flinches over $45 \mathrm{~s}$ for early female TLR4 ${ }^{+/+}$(light gray) and TLR4 cKO mice (light blue). $N$, AUC for summation of flinches demonstrates no significant 
exhibited robust mechanical allodynia that was not significantly different from vehicle-treated controls (Fig. 2H). Surprisingly, the trajectory of allodynia was improved only in females at 5 and 7 weeks after injury, suggesting that peripheral macrophages may be the TLR4-expressing myeloid cells primarily involved in persistent pain in females whereas central microglia may contribute more in males.

Differential turnover rates of peripheral and central myeloid lineage cells allow for specific cKO of TLR4 in microglia alone

In order to fully dissect the contribution of microglial TLR4 to the development of nociceptive sensitization and CRPS signs in the tibial fracture model, we took advantage of differential cell turnover rates between macrophages and microglia (Fig. $3 A$ ) (Parkhurst et al., 2013) to perform targeted KO of TLR4 in microglia alone. To confirm the expression pattern and tamoxifen dependence of the Cx3CR1-Cre ${ }^{E R T 2-e Y F P}$ line (Parkhurst et al., 2013), we first crossed these mice to the Ail4 strain, which has a loxP-flanked STOP cassette preventing transcription of the fluorescent marker, tdTomato. Spinal cord immunohistochemistry clearly demonstrated that tdTomato expression in Cx3CR1$\mathrm{YFP}^{+} \mathrm{Ibal}^{+}$cells required tamoxifen administration (Fig. 3D). We next performed flow cytometry to investigate the persistence of tdTomato expression after tamoxifen administration in myeloid cell populations from CNS or spleen. We found that CNS microglia expressed high levels of tdTomato only after tamoxifen, and this expression persisted for at least $24 \mathrm{~d}$ after tamoxifen (Fig. 3B). In contrast, spleen macrophages expressed tdTomato in a tamoxifen-dependent manner, but this expression was transient and absent at $24 \mathrm{~d}$ after tamoxifen (Fig. $3 C$ ). We next generated a microglial-specific TLR4 cKO using the same Cx3CR1-Cre ${ }^{\text {ERT2-eYFP }}$ strain mouse (Fig. $3 E$ ). Real-time RT-PCR on whole CNS tissue from these mice confirmed a significant, but not complete, loss of Tlr 4 mRNA at 7 and $28 \mathrm{~d}$ after tamoxifen (Fig. $3 F$ ), indicating that CNS cells other than microglia persistently expressed TLR4. In contrast, Tlr4 mRNA in the spleen was not significantly decreased at either time point (Fig. $3 G$ ). Importantly, FACS-sorted microglia were also subjected to realtime RT-PCR analysis for Tlr4 mRNA and levels were undetectable (Fig. 3H). These findings confirm that we generated a microglia-specific TLR4 cKO and moreover that cells other than microglia express TLR4 in the CNS and were unaffected in this mouse.

Early microglia-specific TLR4 KO blocks the transition from acute-to-chronic pain more completely in males

To fully discern the contribution of microglial TLR4 to the postinjury pain trajectory in male and female mice, we dosed TLR4 cKO mice with tamoxifen for $5 \mathrm{~d}$ to knock out TLR4 in myeloid cells. An additional $5 \mathrm{~d}$ was allowed for peripheral myeloid cell turnover, before performing the tibial fracture model of CRPS in these mice (Fig. 4A). At the time of cast removal (3 weeks), all mice developed significant allodynia as evidenced by a significant drop in the mechanical threshold (Fig. 4B). Early TLR4 cKO in males resulted in a complete reversal in allodynia trajectory, with

effect of early TLR4 cKO in females. $n=5$ per group. $\mathbf{0}$, Cumulative duration of nonreflexive behaviors (guarding, jumping, licking) over $45 \mathrm{~s}$ for TLR4 ${ }^{+/+}$(light gray) or early TLR4 CKO (light blue) female mice. $\boldsymbol{P}$, AUC for duration of nonreflexive behaviors demonstrates no change with early TLR4 CKO in females. $n=5$ per group.
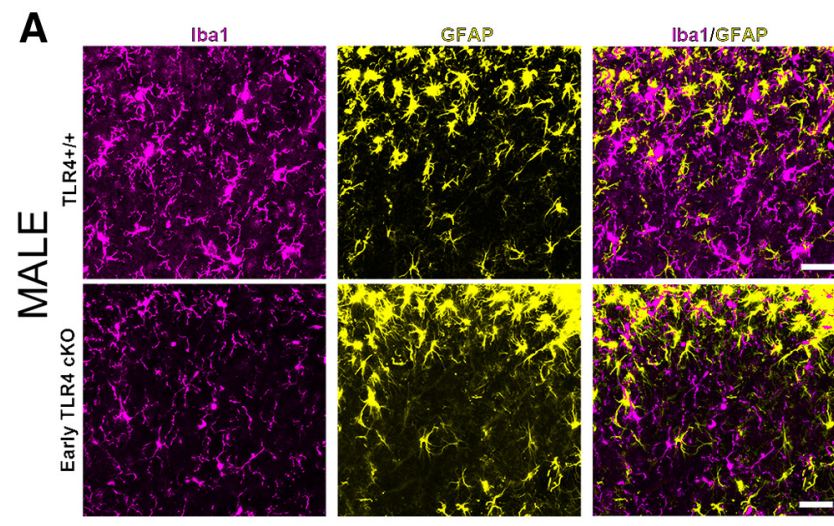

B
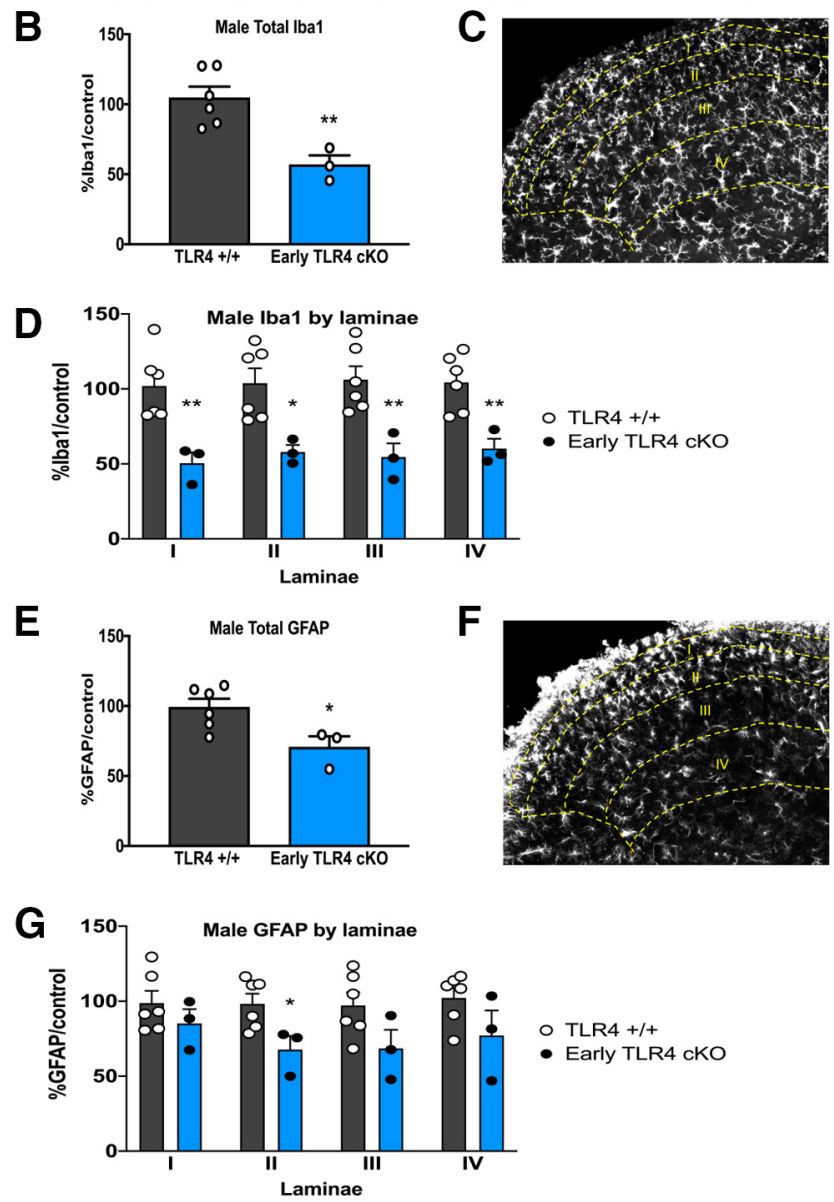

Figure 5. Early TLR4 CKO results in decreased microglial and astrocytic activation in males. A, Immunohistochemistry in TLR4 ${ }^{+/+}$(top row) and TLR4 CKO (bottom row) males showing microglial activation (Iba1/magenta) and astrocyte activation (GFAP/yellow) in the spinal cord dorsal horn 5 weeks after injury. Images taken at $20 \times$. Scale bar, $20 \mu \mathrm{m}$. B, At 5 weeks after injury, early TLR4 CKO results in decreased expression of the microglial marker Iba1 in the entire spinal cord dorsal horn compared with TLR4 ${ }^{+/+}$controls. C, Representative grayscale image showing laminar boundaries with Iba1 staining. $\boldsymbol{D}$, Laminar quantification of microglial Iba1 expression shows significant decreases in lamina I, II, III, and IV in early TLR4 CKO. $\boldsymbol{E}$, At 5 weeks after injury, early TLR4 CKO significantly decreased expression of the astrocyte marker GFAP in the entire spinal cord dorsal horn compared with TLR4 ${ }^{+/+}$controls. $\boldsymbol{F}$, Representative grayscale image showing laminar boundaries with GFAP staining. G, Laminar quantification of astrocytic GFAP expression shows significant decrease only in lamina II in early TLR4 $c K 0 . * p<0.05 ; * * p<0.01$ versus sex-matched TLR4 ${ }^{+/+}$injured mouse by unpaired $t$ test. $n=3-6$ mice per group. Each data point represents the average of 3-6 sections per mouse. Data are mean \pm SEM. 

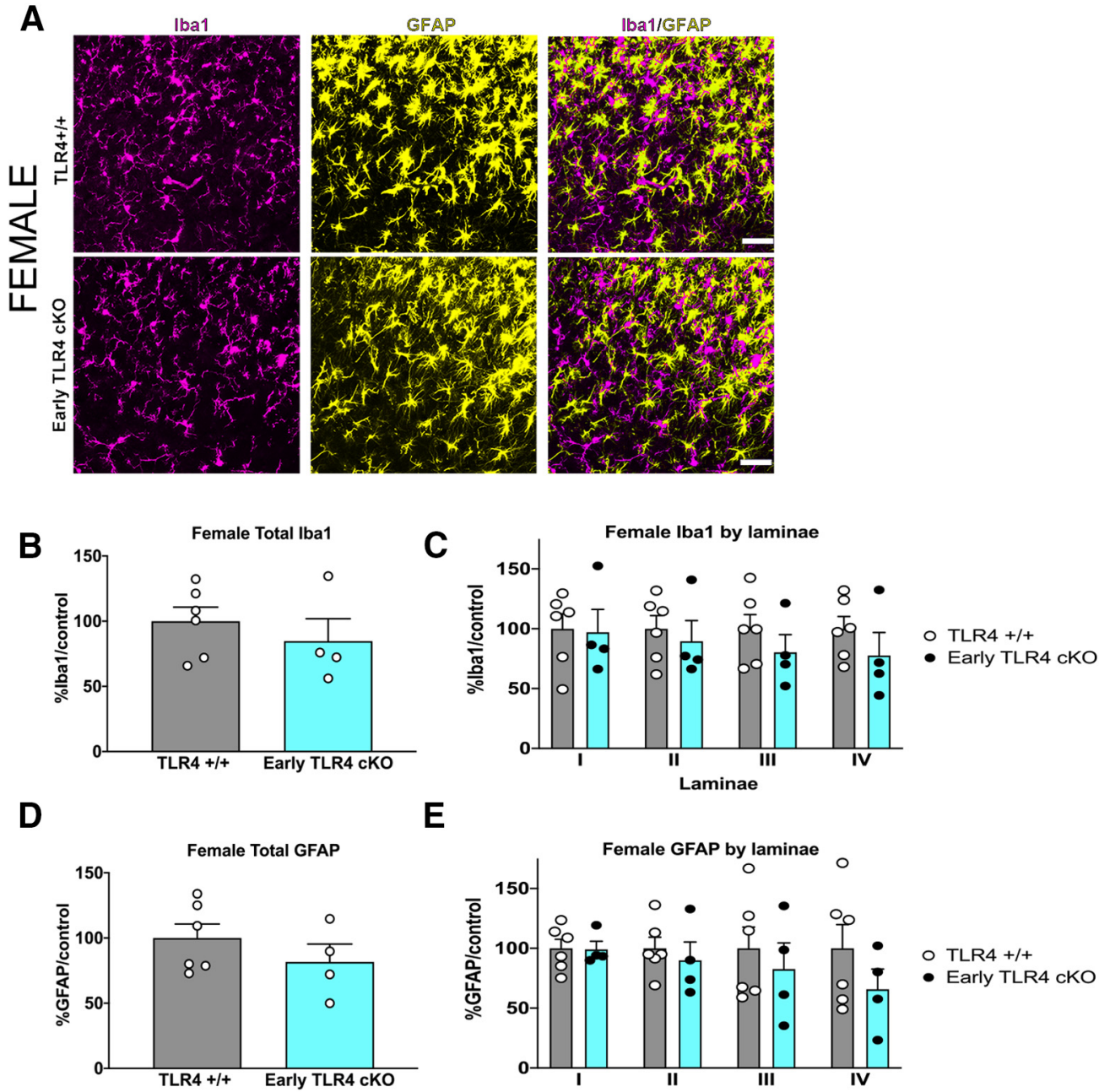

E

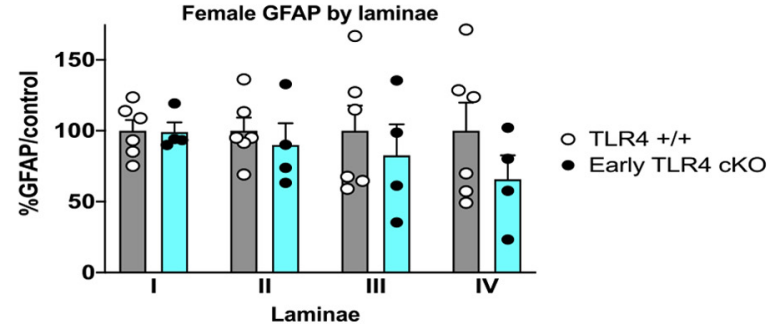

Figure 6. Early TLR4 CKO results in no change in glial activation in females. $A$, Immunohistochemistry in TLR4 ${ }^{+/+}$(top row) and TLR4 cKO (bottom row) females demonstrates similar microglial (Iba1/magenta) and astrocytic (GFAP/yellow) activation in the spinal cord dorsal horn at 5 weeks after injury. Images taken at 20×. Scale bar, $20 \mu \mathrm{m}$. $\boldsymbol{B}$, At 5 weeks after injury, early TLR4 CKO results in no significant change in expression of the microglial marker Iba1 in the entire spinal cord dorsal horn compared with TLR4 ${ }^{+/+}$controls. C, Laminar quantification of microglial Iba1 expression shows no change in any individual lamina in early TLR4 CKO. D, At 5 weeks after injury, early TLR4 cKO does not alter expression of the astrocyte marker GFAP in spinal cord dorsal horn compared with TLR4 ${ }^{+/+}$controls. $E$, Laminar quantification of astrocytic GFAP expression shows no change in any individual lamina in early TLR4 cKO. $n=4-6$ mice per group. Each data point represents the average of 3-6 sections per mouse. Data are mean \pm SEM.

the threshold returning to baseline by 5 weeks after injury, with an effect lasting through 20 weeks (Fig. $4 B$ ). In contrast, early TLR4 cKO in females caused a significant, but more modest, improvement in allodynia starting at 7 weeks after injury that was also sustained through 20 weeks. Weight bearing on the injured paw was significantly improved at 5 weeks after injury in the early TLR4 cKO mice of both sexes, temperature was unchanged, and edema was improved in early TLR4 cKO females only (data not shown). The substantial improvement in mechanical allodynia after early TLR4 cKO in males was accompanied by a significantly increased latency to withdrawal in the hot plate assay (Fig. 4C), while such an effect was not noted in TLR4 cKO females (Fig. 4D). We next scored reflex (flinching) and nonreflexive (guarding, jumping, licking) behaviors on the hot plate (Fig. 4E,F) over $45 \mathrm{~s}$. Early TLR4 cKO in males resulted in a significant decrease in cumulative summation of flinches (Fig. $4 G, H$ ) but had no effect on cumulative duration of nonreflexive behaviors (Fig. 4I,J). Early TLR4 cKO in females had no effect on either cumulative summation of flinches (Fig. $4 K-N$ ) or cumulative duration of nonreflexive behaviors (Fig. 4O,P). In support of a contribution of TLR4 to microglial activation only in males, early TLR4 cKO resulted in decreased morphologic microglial activation and a significant decrease in \% Iba1 in the entire spinal cord dorsal horn in males (Fig. 5A,B). This decrease in Ibal expression in male TLR4 cKO was noted in all superficial laminae (I-IV) of the dorsal horn (Fig. 5C,D), where the majority of primary afferent fibers terminate. Interestingly, astrocytic activation was also suppressed in male TLR4 cKO in the entire spinal dorsal horn (Fig. 5E), but this was driven by a significant decrease in GFAP expression specifically in lamina II (Fig. 5F,G). In contrast, there was no effect of early TLR4 cKO in females on overall dorsal horn Ibal (Fig. 6B) or GFAP expression (Fig. 6D) or within any individual lamina (Ibal, Fig. 6C; and GFAP, Fig. $6 E)$.

\section{Late microglia-specific TLR4 KO results in subtle} improvement in outcomes in both sexes

We next performed "late" TLR4 cKO by dosing tamoxifen for $5 \mathrm{~d}$ from days 13-17 after fracture, which resulted in microglialspecific TLR4 KO by the time of cast removal (Fig. 7A). As expected, at the time of cast removal ( 3 weeks), all mice developed significant allodynia, as evidenced by a significant drop in 
A

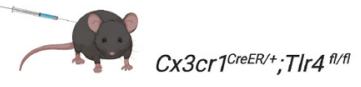

Late Tr/4 conditional knockout

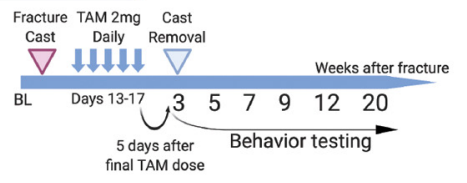

E

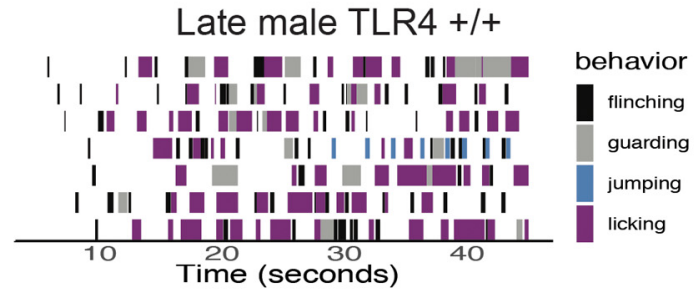

G

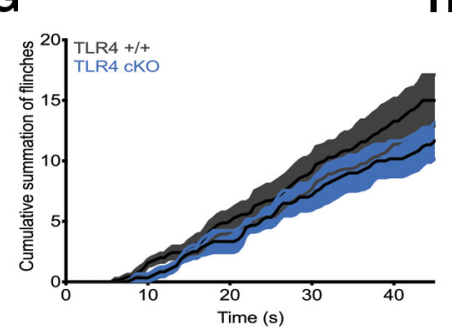

H

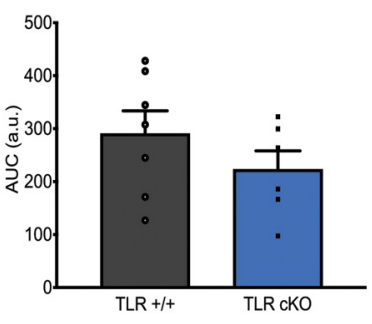

Late female TLR4 +/+

|| || | || | | || || ||||| || behavior

| || |||| || || || || || || ||| | || | | || | |

| | | | | || | || || |||| | | | | || |

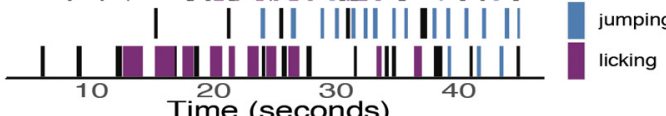

Time (seconds)
M

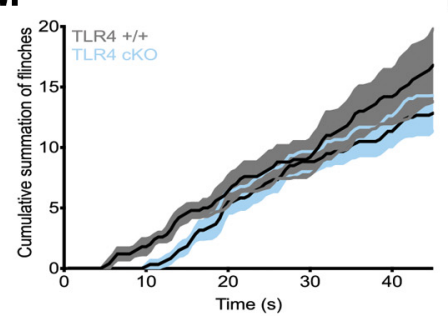

$\mathbf{N}$

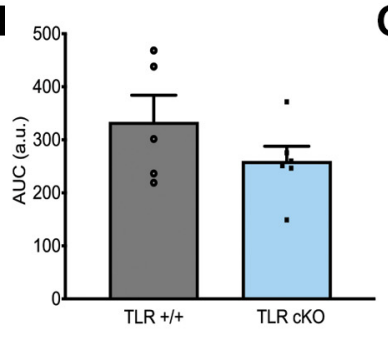

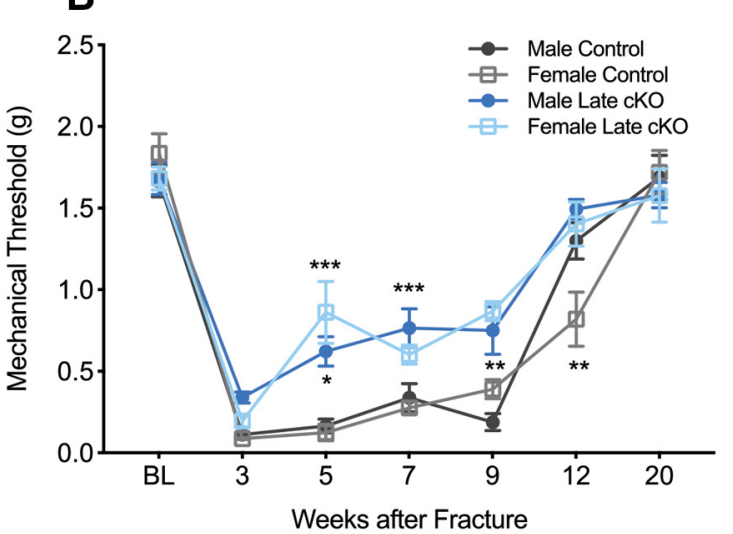

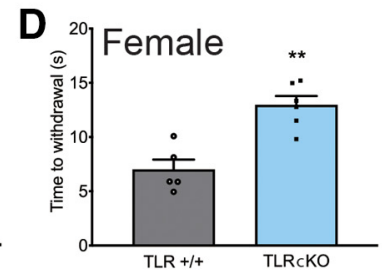

F Late male TLR4 cKO
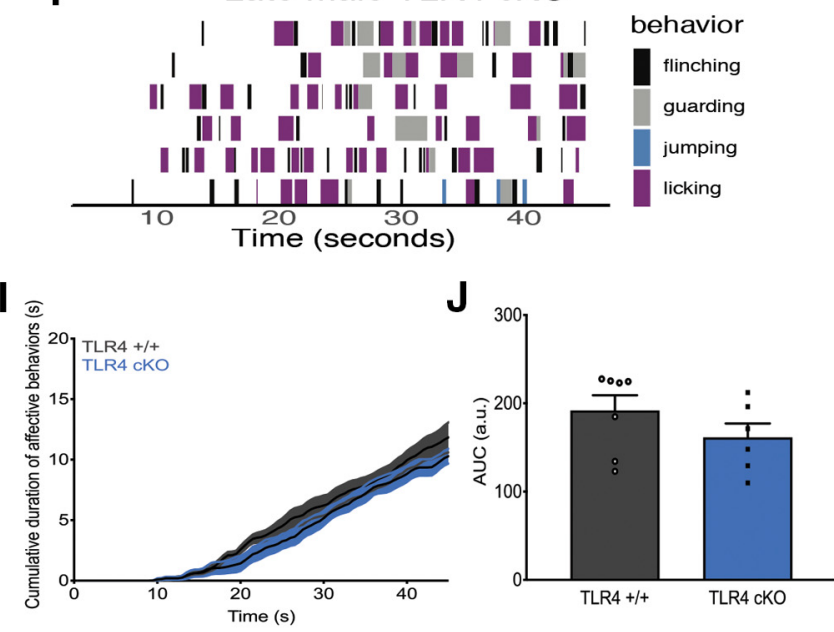

L $\quad$ Late female TLR4 cKO

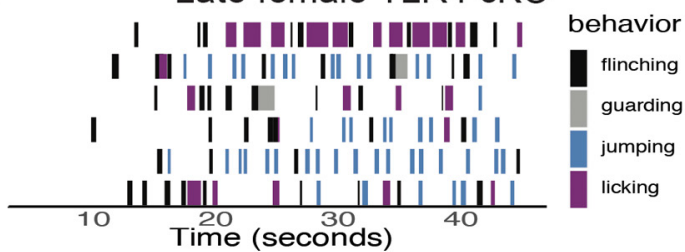

0 斿
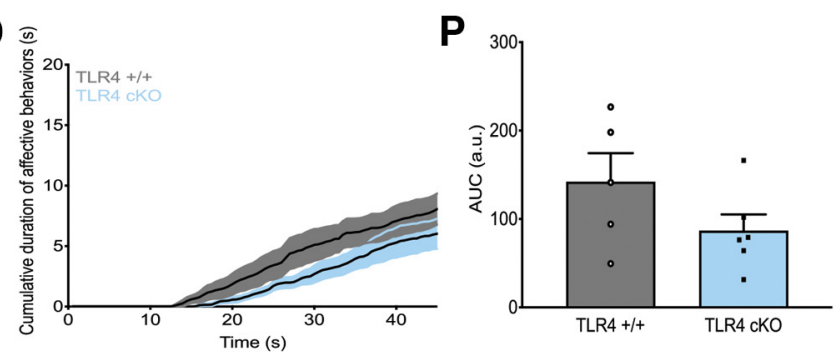

Figure 7. Late CKO of microglial TLR4 has a moderate effect on allodynia and thermal hyperalgesia. $\boldsymbol{A}$, Experimental scheme for late TLR4 K0 in microglia. $\boldsymbol{B}$, Late male and female TLR4 CKO results in significant, but less robust, improvement in persistent allodynia compared with early K0. $n=7-14$ per group. $* p<0.05 ; * * p<0.01 ; * * * p<0.001$ versus sex-matched control by two-way ANOVA with Bonferroni's post-test. Late male (C) and female (D) TLR4 CKO resulted in a significantly increased latency to withdrawal on a $52.5^{\circ} \mathrm{C}$ hot plate. $n=5-7$ per group. $* p<0.05 ; * * p<0.01$ versus sex-matched control by unpaired $t$ test. Mice were placed on an inescapable $52.5^{\circ} \mathrm{C}$ hot plate, and latency to first reflexive response was measured at 5 weeks after fracture. All behavioral responses [flinching (black), guarding (gray), jumping (blue), or licking (purple)] were scored for $45 \mathrm{~s}$ on a $52.5^{\circ} \mathrm{C}$ hot plate for late male TLR4 ${ }^{+/+}(\boldsymbol{E})$ and TLR4 CKO mice $(\boldsymbol{F})$. Each row represents 1 mouse. $n=6$ or 7 per group. $\mathbf{G}$. Cumulative summation of flinches over $45 \mathrm{~s}$ for late male TLR4 ${ }^{+/+}$(dark gray) and TLR4 cKO mice (dark blue). $\boldsymbol{H}$, AUC for summation of flinches demonstrates no significant change in the number of flinches in late male TLR4 CKO. $n=6$ or 7 per group. $* p<0.05$ versus sex-matched control by unpaired $t$ test. $I$, Cumulative duration of nonreflexive behaviors (guarding, jumping, licking) over $45 \mathrm{~s}$ for TLR4 ${ }^{+/+}$(dark gray) or late TLR4 CK0 (dark blue) male mice. J, AUC for duration of nonreflexive behaviors demonstrates no change with late TLR4 CKO in males. $n=5$ per group. All behavioral responses were scored for $45 \mathrm{~s}$ on a $52.5^{\circ} \mathrm{C}$ hot plate for late female TLR4 ${ }^{+/+}(\boldsymbol{K})$ and TLR4 $\mathrm{cKO}$ mice (L). Each row represents 1 mouse. $n=5$ or 6 per group. $\boldsymbol{M}$, Cumulative summation of flinches over $45 \mathrm{~s}$ for late female TLR4 ${ }^{+/+}$(light gray) and TLR4 cKO mice (light blue). $\boldsymbol{N}$, AUC for 

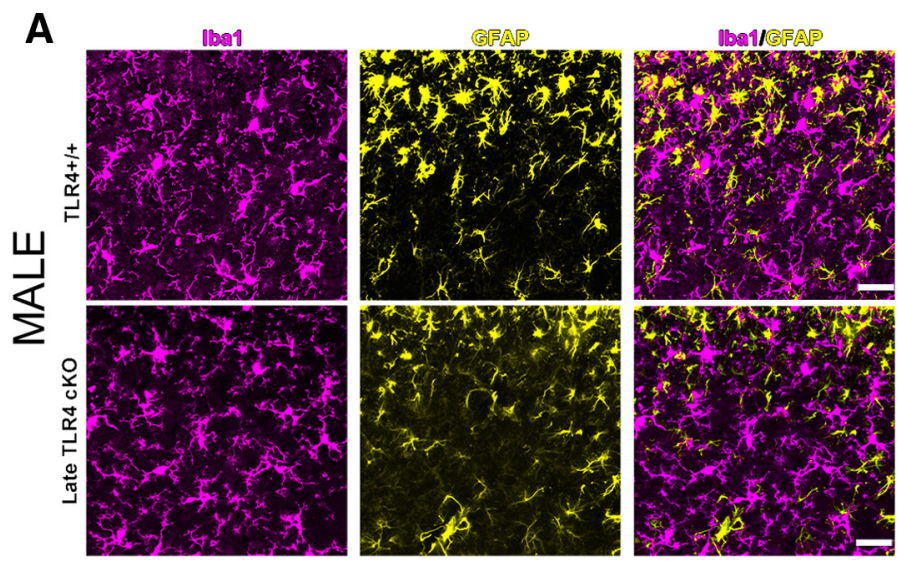

B

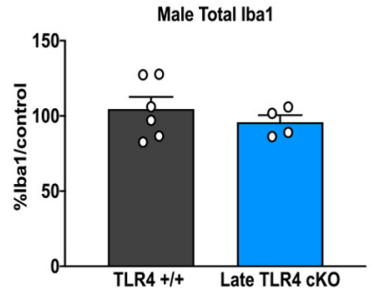

D

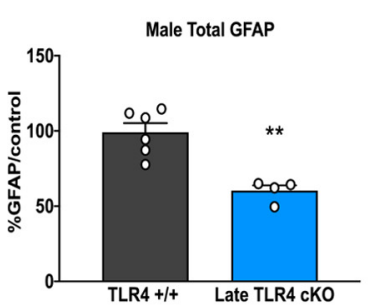

C

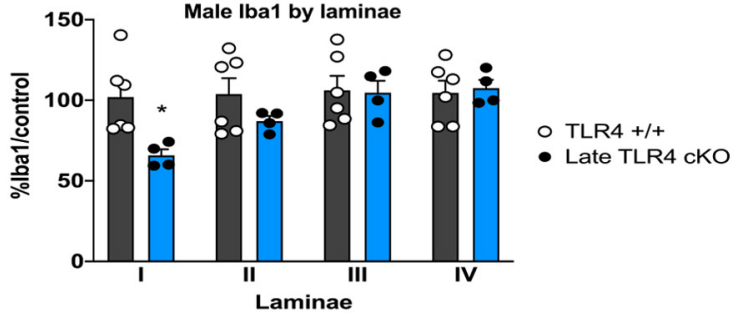

E

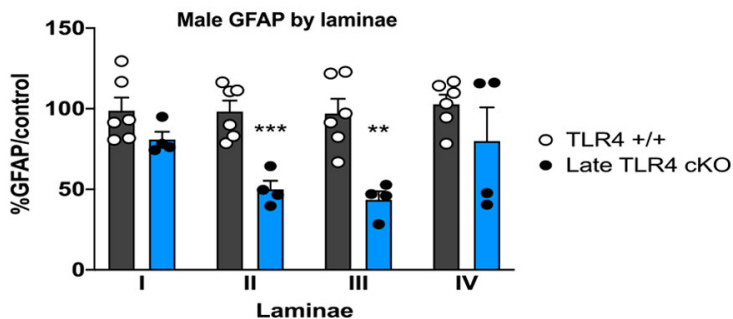

Figure 8. Late TLR4 CKO results in decreased microglial activation in lamina I and decreased astrocytic activation in laminae II and III in males. A, Immunohistochemistry in TLR4 ${ }^{+/+}$(top row) and TLR4 CKO (bottom row) males showing microglial activation (Iba1/magenta) and astrocyte activation (GFAP/yellow) in the spinal cord dorsal horn 5 weeks after injury in late TLR4 cK0 mice. Images taken at 20×. Scale bar, $20 \mu \mathrm{m} . \boldsymbol{B}$, At 5 weeks after injury, late TLR4 cK0 did not alter the overall expression of the microglial marker Iba1 in spinal cord dorsal horn compared with TLR4 ${ }^{+/+}$controls. C, Laminar quantification of microglial Iba1 expression shows significant decreases only in lamina I in late TLR4 CKO. D, At 5 weeks after injury, late TLR4 cK0 significantly reduced expression of the astrocyte marker GFAP in the entire spinal cord dorsal horn compared with TLR4 ${ }^{+/+}$controls. $\boldsymbol{E}$, Laminar quantification of astrocytic GFAP expression shows significant decreases in lamina II and III in late TLR4 CKO. $* p<0.05 ; * * p<0.01 ; * * * p<0.001$ versus sex-matched TLR4 ${ }^{+/+}$injured mouse by unpaired $t$ test. $n=4-6$ mice per group. Each data point represents the average of 3-6 sections per mouse. Data are mean \pm SEM.

the mechanical threshold (Fig. $7 B$ ). In contrast to early TLR4 cKO, late TLR4 cKO resulted in a modest improvement in allodynia in males and females from weeks 5 to 9 after injury (Fig. $7 B)$. Late TLR4 cKO resulted in improved weight bearing on the injured paw, and normalization of temperature and edema in mice of both sexes (data not shown). Late TLR4 cKO in males and females significantly increased latency to withdrawal in the hot plate assay (Fig. $7 C, D$ ). We again scored reflex (flinching) and nonreflexive (guarding, jumping, licking) behaviors on the hot plate (Fig. $7 E-P$ ) over 45 s. Late TLR 4 cKO did not alter the cumulative summation of flinches in males (Fig. 7G,H) or females (Fig. $7 M, N$ ) or cumulative duration of nonreflexive behaviors in either sex (Fig. 7I,J,O,P). Late TLR4 cKO did not change microglial morphology or \% Ibal in the entire spinal

\footnotetext{
summation of flinches demonstrates no significant effect of late TLR4 cK0 in females. $n=5$ or 6 per group. $\mathbf{0}$, Cumulative duration of nonreflexive behaviors (guarding, jumping, licking) over $45 \mathrm{~s}$ for TLR4 ${ }^{+/+}$(light gray) or late TLR4 cKO (light blue) female mice. P, AUC for duration of nonreflexive behaviors demonstrates no change with late TLR4 CKO in females. $n=5$ or 6 per group.
}

cord dorsal horn in males (Fig. $8 A, B$ ) or females (Fig. 9A,B) but did significantly decrease Ibal expression in lamina $I$ only in males (Fig. 8C) and females (Fig. 9C). Additionally, we observed decreased astrocytic GFAP expression in the entire spinal cord dorsal horn after late TLR4 cKO in males (Fig. 8D) and females (Fig. 9D). More specifically, this was noted in lamina II and III (Fig. $8 E$ ) in males and in lamina I, II, and III (Fig. 9E) in females.

\section{Discussion}

In this study, we evaluated the location-, time-, and sex-dependent contributions of myeloid cell TLR4 to the acute-to-chronic pain transition in the clinically relevant tibial fracture model of CRPS. We provide evidence that TLR4 contributes to persistent allodynia and thermal hyperalgesia in both males and females; however, the relative contribution of major TLR4-expressing cells is sex-dependent. We demonstrate that both sexes exhibit improvement in allodynia when treated with a systemically available TLR4 antagonist. This effect is mimicked with a peripheral macrophage CCR2 receptor antagonist in females only, and central TLR4 antagonism in males only. Furthermore, we show that early knockdown of microglial TLR4 completely reverses the 
A

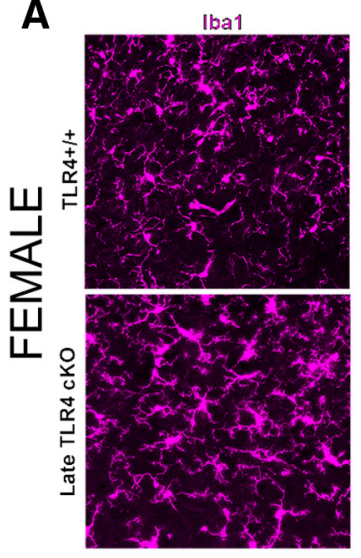

GEAP

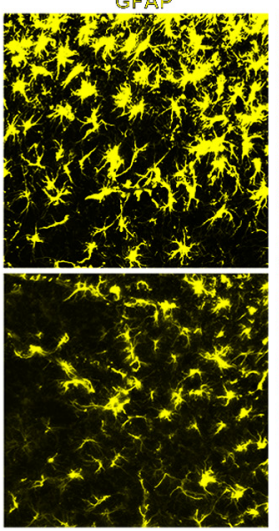

Iba1/GFAP

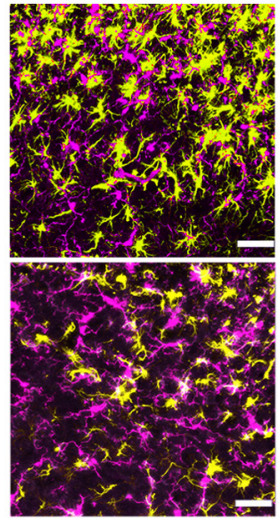

B

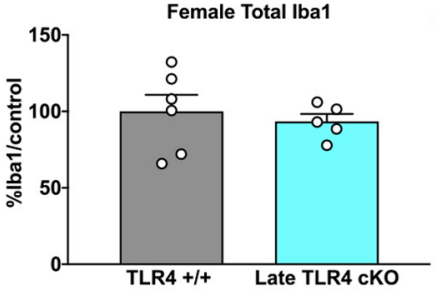

D

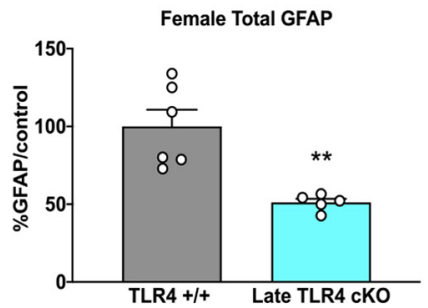

C

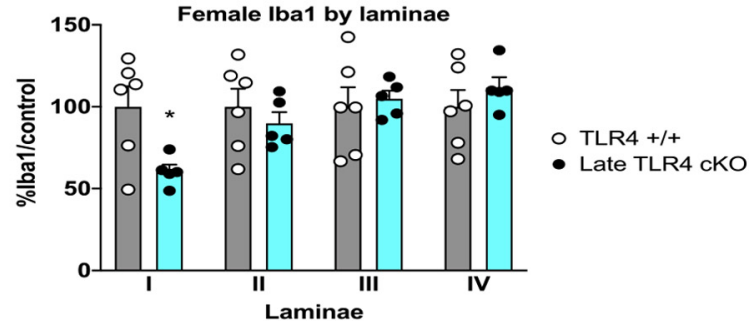

E

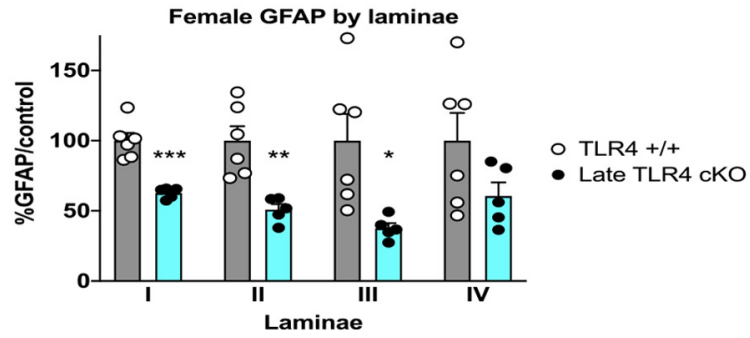

Figure 9. Late TLR4 CKO results in decreased microglial activation in lamina I and decreased astrocyte activation throughout the dorsal horn in females. $\boldsymbol{A}$, Immunohistochemistry in $\mathrm{TLR}^{+/+}$(top row) and TLR4 CKO (bottom row) females demonstrates similar microglial activation (lba1/magenta), but reduced astrocyte activation (GFAP/yellow) in the spinal cord dorsal horn 5 weeks after injury in late TLR4 cK0 mice. Images taken at 20×. Scale bar, $20 \mu \mathrm{m}$. B, At 5 weeks after injury, late TLR4 cK0 did not alter the overall expression of the microglial marker Iba1 in spinal cord dorsal horn compared with TLR4 ${ }^{+/+}$controls. C, Laminar quantification of microglial Iba1 expression shows significant decreases only in lamina I in late TLR4 cKO. D, At 5 weeks after injury, late TLR4 CKO reduced expression of the astrocyte marker GFAP in the entire spinal cord dorsal horn compared with TLR4 ${ }^{+/+}$controls. $E$, Laminar quantification of astrocytic GFAP expression shows significant decreases in lamina I, II, and III in late TLR4 CKO. $* p<0.05 ; * * p<0.01 ; * * * p<0.001$ versus sex-matched TLR4 ${ }^{+/+}$injured mouse by unpaired $t$ test. $n=5$ or 6 mice per group. Each data point represents the average of 3-6 sections per mouse. Data are mean \pm SEM.

trajectory of mechanical allodynia, lessens hot plate reflexive responses, and suppresses microglial activation in males, with a minimal effect in females. Finally, we demonstrate that, for both sexes, late knockdown of microglial TLR4 produces only minimal improvement in allodynia but does suppress astrocytic GFAP at 5 weeks, suggesting that downstream cellular or molecular TLR4-independent events such as synaptic plasticity (Liddelow et al., 2017), may have already been triggered and contribute more to the chronic/late phase of CRPS. Together, our findings highlight the complex influence of TLR4 in the acuteto-chronic pain transition.

Perhaps our most striking finding is the apparent involvement of TLR4 in both male and female CRPS progression. While previous studies have suggested that microglial TLR4 is a predominantly male mediator of pain (Sorge et al., 2011, 2015), we used complementary pharmacologic and transgenic approaches to establish that the contribution of TLR4 is perhaps more nuanced than previously thought. Important to this discussion is the fact that TLR4 is expressed on multiple cell types, including peripheral and central myeloid-lineage cells, neurons, astrocytes, and even endothelial cells (Grace et al., 2014b; Ji et al., 2016). To our knowledge, all previous studies used germline TLR4 dominant negative or KO mice in which TLR4 is lost from all cell types in a constitutive manner. These strains do not allow for determination of cell-type specificity, time-dependent effects, or possible compensatory changes that may occur during development. In comparison, the Cx3CR1-Cre ERT2-eYFP mouse offers a unique opportunity to perform microglia-specific KO of TLR4 with temporal control. The mouse was originally developed to clarify whether microglia consist of a separate yolk sac-derived myeloid cell population that renews from CNS resident cells (Parkhurst et al., 2013; Bruttger et al., 2015). These mice have been used to target microglia for gene manipulation by taking advantage of the low turnover rates of microglia (Lawson et al., 1992) compared with peripheral myeloid cells. For example, Mousseau et al. (2018) used this approach to perform targeted microglial depletion of the pannexin-1 channel and demonstrated its importance in IL- $1 \beta$ release in a mouse arthritis model. There are, however, caveats to the use of these mice, with leakiness to other cell types and constitutive activation of the Cre recombinase reported (Zhao et al., 2019). Therefore, we undertook an extensive series of experiments using the Ail4 reporter 
line before initiating our behavioral experiments. As a result, we are confident that the TLR $4 \mathrm{cKO}$ was limited to microglia, after time for turnover of peripheral myeloid-lineage cells. It should be noted, however, that with the late TLR4 cKO, there is an inherent, transient loss of macrophage TLR4 before cast removal at 3 weeks because the tamoxifen was given on days 13-17.

The peripheral versus central nature of contributory TLR4expressing cells has been previously assessed using location-specific administration of TLR4 antagonists. Sorge et al. (2011) found that intrathecal LPS, the classic TLR agonist, triggered TLR4-dependent nociceptive responses only in male CD-1 mice, and that LPS-rs, a TLR4 antagonist, given intrathecally improved male, but not female, allodynia after spared nerve injury. The interpretation of these findings was that central TLR4-expressing microglia were responsible for the effect; however, the contribution of neuronal or astrocytic TLR4 could not be excluded. In contrast, Woller et al. (2016) found that intrathecal LPS did cause allodynia in female C57Bl/6 mice; however, this effect was not blocked by the TLR4 antagonist, TAK242, or by constitutive TLR4 KO indicating that LPS-induced allodynia may occur through other pathways, such as the noncanonical inflammasome (Kayagaki et al., 2013). Furthermore, in the same study, allodynia in Phase 3 of the formalin model was attenuated by TAK242 in both sexes. In agreement, Agalave et al. (2014) demonstrated that intrathecal administration of the TLR agonist, HMGB1, produced allodynia in both male and female C57Bl/6 mice in a TLR4-dependent manner, and that neutralizing HMGB1 reduced allodynia in a model of arthritis in both sexes. The tibial fracture model of CRPS, used in the current study, engages both neuropathic and inflammatory mechanisms (Birklein et al., 2018; Clark et al., 2018), and we too found that systemic TLR4 antagonism in this model improved pain trajectory in both male and female $\mathrm{C} 57 \mathrm{Bl} / 6 \mathrm{~J}$ mice. Together, the contribution of myeloid-lineage TLR4 may therefore vary depending on the strain of mouse used and the extent of the inflammatory component of the pain model being studied.

Microglial activation is not an all-or-none phenomenon (Ransohoff, 2016), and it remains unclear whether triggering microglia via TLR4 or other known activating receptors important in pain, such as CSF1 (Guan et al., 2016) or P2X4 (Tsuda et al., 2003), all result in the same phenotype. It is known, however, that microglia in the spinal cord express higher levels of markers, such as CD11b and Ibal after injury in a variety of pain models in both sexes (Sorge et al., 2015; Taves et al., 2016; FernandezZafra et al., 2019) and that microglial activation can be dissociated from pain (Colburn et al., 1997). Importantly, depletion of microglia early after spinal nerve transection results in improvement in allodynia in males and females (Peng et al., 2016), suggesting that microglia contribute to the initiation of pain in both sexes. Taves et al. (2016) administered the myeloid cell p38 mitogen-activated protein kinase inhibitor, skepinone, intrathecally, systemically, or perineurally in the chronic constriction model. In agreement with our own findings, an improvement in allodynia was confirmed in both sexes with peripheral TAK242 administration, but only in males with central administration, implying that the effect was related to inhibition of central microglia in males and peripheral myeloid-lineage cells in females. Interestingly, our colleagues (Michael Burton, personal communication) used a novel TLR4 mouse to reactivate TLR4 only in Nav1.8-expressing primary afferent neurons and found that this was sufficient to drive pain behaviors in female, but not male, mice. This elegant study highlights the possibility that other peripheral TLR4-expressing cells, beyond the myeloid lineage, may additionally have contributed to the clear improvement in allodynia we noted with a systemically administered TLR4 antagonist in females.

Our findings also have important clinical implications for CRPS, a condition with a female predominance of up to 4:1 (Sandroni et al., 2003). One clinical trial exists to study the effects of low-dose naltrexone on pain in CRPS (NCT02502162). Naltrexone is a centrally acting opioid antagonist which, at low doses, primarily antagonizes TLR4 (Wang et al., 2016) and has analgesic properties in various preclinical pain models (Hutchinson et al., 2008; Kao et al., 2015). Our data suggest that early treatment, or perhaps pretreatment in the case of high-risk patients undergoing elective surgery, with TLR4-modulating agents such as low-dose naltrexone may be more beneficial than late treatment. Importantly, these agents may be effective both for male and female patients, with centrally acting agents perhaps providing greater benefit in males than in females.

Here we present several unique and important analyses of sex-dependent and spatiotemporal involvement of TLR4 in CRPS progression from the acute to chronic phase. Overall, we find that the contribution of TLR4 is time- and microglia-dependent in both sexes; however, females also rely on peripheral myeloid-lineage (or other TLR4-expressing) cells to trigger chronic pain.

\section{References}

Agalave NM, Larsson M, Abdelmoaty S, Su J, Baharpoor A, Lundback P, Palmblad K, Andersson U, Harris H, Svensson CI (2014) Spinal HMGB1 induces TLR4-mediated long-lasting hypersensitivity and glial activation and regulates pain-like behavior in experimental arthritis. Pain 155:18021813.

Birklein F, Ibrahim A, Schlereth T, Kingery WS (2018) The rodent tibia fracture model: a critical review and comparison with the complex regional pain syndrome literature. J Pain 19:e1101-e1102.

Bruttger J, Karram K, Wortge S, Regen T, Marini F, Hoppmann N, Klein M, Blank T, Yona S, Wolf Y, Mack M, Pinteaux E, Muller W, Zipp F, Binder H, Bopp T, Prinz M, Jung S, Waisman A (2015) Genetic cell ablation reveals clusters of local self-renewing microglia in the mammalian central nervous system. Immunity 43:92-106.

Chaplan SR, Bach FW, Pogrel JW, Chung JM, Yaksh TL (1994) Quantitative assessment of tactile allodynia in the rat paw. J Neurosci Methods 53:5563.

Clark JD, Tawfik VL, Tajerian M, Kingery WS (2018) Autoinflammatory and autoimmune contributions to complex regional pain syndrome. Mol Pain 14:1744806918799127.

Colburn RW, DeLeo JA, Rickman AJ, Yeager MP, Kwon P, Hickey WF (1997) Dissociation of microglial activation and neuropathic pain behaviors following peripheral nerve injury in the rat. J Neuroimmunol 79:163-175.

Corder G, Tawfik VL, Wang D, Sypek EI, Low SA, Dickinson JR, Sotoudeh C, Clark JD, Barres BA, Bohlen CJ, Scherrer G (2017) Loss of mu opioid receptor signaling in nociceptors, but not microglia, abrogates morphine tolerance without disrupting analgesia. Nat Med 23:164-173.

Cropper HC, Johnson EM, Haight ES, Cordonnier SA, Chaney AM, Forman TE, Biswal A, Stevens MY, James ML, Tawfik VL (2019) Longitudinal translocator protein-18 $\mathrm{kDa}$-positron emission tomography imaging of peripheral and central myeloid cells in a mouse model of complex regional pain syndrome. Pain 160:2136-2148.

Fernandez-Zafra T, Gao T, Jurczak A, Sandor K, Hore Z, Agalave NM, Su J, Estelius J, Lampa J, Hokfelt T, Wiesenfeld-Hallin Z, Xu X, Denk F, Svensson CI (2019) Exploring the transcriptome of resident spinal microglia after collagen antibody-induced arthritis. Pain 160:224-236.

Gallagher JJ, Tajerian M, Guo T, Shi X, Li W, Zheng M, Peltz G, Kingery WS, Clark JD (2013) Acute and chronic phases of complex regional pain syndrome in mice are accompanied by distinct transcriptional changes in the spinal cord. Mol Pain 9:40.

Grace PM, Hutchinson MR, Maier SF, Watkins LR (2014a) Pathological pain and the neuroimmune interface. Nat Rev Immunol 14:217-231. 
Grace PM, Ramos KM, Rodgers KM, Wang X, Hutchinson MR, Lewis MT, Morgan KN, Kroll JL, Taylor FR, Strand KA, Zhang Y, Berkelhammer D, Huey MG, Greene LI, Cochran TA, Yin H, Barth DS, Johnson KW, Rice KC, Maier SF, et al. (2014b) Activation of adult rat CNS endothelial cells by opioid-induced toll-like receptor 4 (TLR4) signaling induces proinflammatory, biochemical, morphological, and behavioral sequelae. Neuroscience 280:299-317.

Guan Z, Kuhn JA, Wang X, Colquitt B, Solorzano C, Vaman S, Guan AK, Evans-Reinsch Z, Braz J, Devor M, Abboud-Werner SL, Lanier LL, Lomvardas S, Basbaum AI (2016) Injured sensory neuron-derived CSF1 induces microglial proliferation and DAP12-dependent pain. Nat Neurosci 19:94-101.

Guo TZ, Wei T, Li WW, Li XQ, Clark JD, Kingery WS (2014) Immobilization contributes to exaggerated neuropeptide signaling, inflammatory changes, and nociceptive sensitization after fracture in rats. J Pain 15:1033-1045.

Hua F, Tang H, Wang J, Prunty MC, Hua X, Sayeed I, Stein DG (2015) TAK242 , an antagonist for Toll-like receptor 4, protects against acute cerebral ischemia/reperfusion injury in mice. J Cereb Blood Flow Metab 35:536542.

Hutchinson MR, Zhang Y, Brown K, Coats BD, Shridhar M, Sholar PW, Patel SJ, Crysdale NY, Harrison JA, Maier SF, Rice KC, Watkins LR (2008) Non-stereoselective reversal of neuropathic pain by naloxone and naltrexone: involvement of toll-like receptor 4 (TLR4). Eur J Neurosci 28:20-29.

Institute of Medicine (2011) Relieving pain in America: a blueprint for transforming prevention, care, education and research. Washington, DC: The National Academies Press.

Ji RR, Chamessian A, Zhang YQ (2016) Pain regulation by non-neuronal cells and inflammation. Science 354:572-577.

Kao JH, Gao MJ, Yang PP, Law PY, Loh HH, Tao PL (2015) Effect of naltrexone on neuropathic pain in mice locally transfected with the mutant muopioid receptor gene in spinal cord. Br J Pharmacol 172:630-641.

Kayagaki N, Wong MT, Stowe IB, Ramani SR, Gonzalez LC, AkashiTakamura S, Miyake K, Zhang J, Lee WP, Muszyński A, Forsberg LS, Carlson RW, Dixit VM (2013) Noncanonical inflammasome activation by intracellular LPS independent of TLR4. Science 341:1246-1249.

Lawson LJ, Perry VH, Gordon S (1992) Turnover of resident microglia in the normal adult mouse brain. Neuroscience 48:405-415.

Liddelow SA, Guttenplan KA, Clarke LE, Bennett FC, Bohlen CJ, Schirmer L, Bennett ML, Munch AE, Chung WS, Peterson TC, Wilton DK, Frouin A, Napier BA, Panicker N, Kumar M, Buckwalter MS, Rowitch DH, Dawson VL, Dawson TM, Stevens B, et al. (2017) Neurotoxic reactive astrocytes are induced by activated microglia. Nature 541:481-487.

Livak KJ, Schmittgen TD (2001) Analysis of relative gene expression data using real-time quantitative PCR and the 2(-delta delta $\mathrm{C}(\mathrm{T})$ ) method. Methods 25:402-408.

McAlees JW, Whitehead GS, Harley IT, Cappelletti M, Rewerts CL, Holdcroft AM, Divanovic S, Wills-Karp M, Finkelman FD, Karp CL, Cook DN (2015) Distinct Tlr4-expressing cell compartments control neutrophilic and eosinophilic airway inflammation. Mucosal Immunol 8:863-873.

Mousseau M, Burma NE, Lee KY, Leduc-Pessah H, Kwok CH, Reid AR, O'Brien M, Sagalajev B, Stratton JA, Patrick N, Stemkowski PL, Biernaskie J, Zamponi GW, Salo P, McDougall JJ, Prescott SA, Matyas JR, Trang T (2018) Microglial pannexin-1 channel activation is a spinal determinant of joint pain. Sci Adv 4:eaas9846.

Parkhurst CN, Yang G, Ninan I, Savas JN, Yates JR 3rd, Lafaille JJ, Hempstead BL, Littman DR, Gan WB (2013) Microglia promote learning-dependent synapse formation through brain-derived neurotrophic factor. Cell 155:1596-1609.
Peng J, Gu N, Zhou L, B Eyo U, Murugan M, Gan WB, Wu LJ (2016) Microglia and monocytes synergistically promote the transition from acute to chronic pain after nerve injury. Nat Commun 7:12029.

Raghu H, Lepus CM, Wang Q, Wong HH, Lingampalli N, Oliviero F, Punzi L, Giori NJ, Goodman SB, Chu CR, Sokolove JB, Robinson WH (2017) CCL2/CCR2, but not CCL5/CCR5, mediates monocyte recruitment, inflammation and cartilage destruction in osteoarthritis. Ann Rheum Dis 76:914-922.

Ransohoff RM (2016) A polarizing question: do M1 and M2 microglia exist? Nat Neurosci 19:987-991.

Sandroni P, Benrud-Larson LM, McClelland RL, Low PA (2003) Complex regional pain syndrome type I: incidence and prevalence in Olmsted County, a population-based study. Pain 103:199-207.

Schindelin J, Arganda-Carreras I, Frise E, Kaynig V, Longair M, Pietzsch T, Preibisch S, Rueden C, Saalfeld S, Schmid B, Tinevez JY, White DJ, Hartenstein V, Eliceiri K, Tomancak P, Cardona A (2012) Fiji: an opensource platform for biological-image analysis. Nat Methods 9:676-682.

Sorge RE, LaCroix-Fralish ML, Tuttle AH, Sotocinal SG, Austin JS, Ritchie J, Chanda ML, Graham AC, Topham L, Beggs S, Salter MW, Mogil JS (2011) Spinal cord Toll-like receptor 4 mediates inflammatory and neuropathic hypersensitivity in male but not female mice. J Neurosci 31:15450-15454.

Sorge RE, Martin LJ, Isbester KA, Sotocinal SG, Rosen S, Tuttle AH, Wieskopf JS, Acland EL, Dokova A, Kadoura B, Leger P, Mapplebeck JC, McPhail M, Delaney A, Wigerblad G, Schumann AP, Quinn T, Frasnelli J, Svensson CI, Sternberg WF, et al. (2014) Olfactory exposure to males, including men, causes stress and related analgesia in rodents. Nat Methods 11:629-632.

Sorge RE, Mapplebeck JC, Rosen S, Beggs S, Taves S, Alexander JK, Martin LJ, Austin JS, Sotocinal SG, Chen D, Yang M, Shi XQ, Huang H, Pillon NJ, Bilan PJ, Tu Y, Klip A, Ji RR, Zhang J, Salter MW, et al. (2015) Different immune cells mediate mechanical pain hypersensitivity in male and female mice. Nat Neurosci 18:1081-1083.

Tanga FY, Nutile-McMenemy N, DeLeo JA (2005) The CNS role of Toll-like receptor 4 in innate neuroimmunity and painful neuropathy. Proc Natl Acad Sci USA 102:5856-5861.

Taves S, Berta T, Liu DL, Gan S, Chen G, Kim YH, Van de Ven T, Laufer S, Ji RR (2016) Spinal inhibition of p38 MAP kinase reduces inflammatory and neuropathic pain in male but not female mice: sex-dependent microglial signaling in the spinal cord. Brain Behav Immun 55:70-81.

Tsuda M, Shigemoto-Mogami Y, Koizumi S, Mizokoshi A, Kohsaka S, Salter MW, Inoue K (2003) P2X4 receptors induced in spinal microglia gate tactile allodynia after nerve injury. Nature 424:778-783.

Wang X, Zhang Y, Peng Y, Hutchinson MR, Rice KC, Yin H, Watkins LR (2016) Pharmacological characterization of the opioid inactive isomers $(+)$-naltrexone and (+)-naloxone as antagonists of toll-like receptor 4. Br J Pharmacol 173:856-869.

Woller SA, Ravula SB, Tucci FC, Beaton G, Corr M, Isseroff RR, Soulika AM, Chigbrow M, Eddinger KA, Yaksh TL (2016) Systemic TAK-242 prevents intrathecal LPS evoked hyperalgesia in male, but not female mice and prevents delayed allodynia following intraplantar formalin in both male and female mice: the role of TLR4 in the evolution of a persistent pain state. Brain Behav Immun 56:271-280.

Xanthos DN, Sandkuhler J (2014) Neurogenic neuroinflammation: inflammatory CNS reactions in response to neuronal activity. Nat Rev Neurosci $15: 43-53$

Zhao XF, Alam MM, Liao Y, Huang T, Mathur R, Zhu X, Huang Y (2019) Targeting microglia using Cx3cr1-Cre lines: revisiting the specificity. eneuro 6:ENEURO.0114-19.2019. 